Mon. Not. R. Astron. Soc. 000, 1 15() Printed 25 July $2017 \quad$ (MN LATEX style file v2.2)

\title{
Estimation of mass outflow rates from dissipative accretion disc around rotating black holes
}

\author{
Ramiz Aktar ${ }^{1 \star}$, Santabrata Das ${ }^{1}$, Anuj Nandi ${ }^{2}$, H. Sreehari ${ }^{2,3}$ * \\ ${ }^{1}$ Indian Institute of Technology Guwahati, Guwahati, 781039, India \\ ${ }^{2}$ Space Astronomy Group, ISITE Campus, ISRO Satellite Centre, Outer Ring Road, Marathahalli, Bangalore, 560037, India \\ ${ }^{3}$ Indian Institute of Science, Bangalore, 560012, India
}

Accepted . Received ; in original form

\begin{abstract}
We study the properties of the dissipative accretion flow around rotating black holes in presence of mass loss. We obtain the complete set of global inflow-outflow solutions in the steady state by solving the underlying conservation equations selfconsistently. We observe that global inflow-outflow solutions are not the isolated solution, instead such solutions are possible for wide range of inflow parameters. Accordingly, we identify the boundary of the parameter space for outflows, spanned by the angular momentum $\left(\lambda_{\text {in }}\right)$ and the energy $\left(\mathcal{E}_{\text {in }}\right)$ at the inner sonic point $\left(x_{\text {in }}\right)$, as function of the dissipation parameters and find that parameter space gradually shrinks with the increase of dissipation rates. Further, we examine the properties of the outflow rate $R_{\dot{m}}$ (defined as the ratio of outflow to inflow mass flux) and ascertain that dissipative processes play the decisive role in determining the outflow rates. We calculate the limits on the maximum outflow rate $\left(R_{\dot{m}}^{\max }\right)$ in terms of viscosity parameter $(\alpha)$ as well as black hole spin $\left(a_{k}\right)$ and obtain the limiting range as $3 \% \leqslant R_{\dot{m}}^{\max } \leqslant 19 \%$. Moreover, we calculate the viable range of $\alpha$ that admits the coupled inflow-outflow solutions and find that $\alpha \lesssim 0.25$ for $R_{\dot{m}} \neq 0$. Finally, we discuss the observational implication of our formalism to infer the spin of the black holes. Towards this, considering the highest observed QPO frequency of black hole source GRO J1655-40 ( 450 $\mathrm{Hz}$ ), we constrain the spin value of the source as $a_{k} \geqslant 0.57$.
\end{abstract}

Key words: accretion, accretion disc - black hole physics - shock waves - ISM: jets and outflows-X-rays: binaries.

\section{INTRODUCTION}

In accretion system around black holes, jets and outflows play an essential role and their existence is realized both in observations and simulations (Mirabel et al. 1992, Mirabel \& Rodriguez | 1994. Hjellming \& Rupen |1995; Ferrari 1998. Mirabel \& Rodriguez 1998; Junor et al. 1999; Cheung 2002 Mirabel 2003| Fender et al. 2009; Miller et al. 2012, Mezcua et al. |2013; Das et al.| 2014 | Okuda \& Das |2015; Mezcua et al. |2015, Fender \& Munoz-Darias |2016). Since black holes do not have any hard boundary, the outflows and jets must be originated from the accretion disc and observations also indeed confirm the disc-jet connection (Feroci et al. 1999

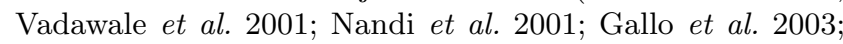
Miller et al. 2012, Miller-Jones et al. 2012 | Corbel et al. 2013 Radhika \& Nandi 2014; Radhika et al. 2016a). Earlier the- oretical works of Penrose (1969) and Blandford \& Znajek (1977) suggested that the powerful jets are originated due to the rotation of the black hole. However, conflicting claims were made from the observational front. Steiner et al. (2013); McClintock et al. (2014) reported the significant evidences of positive correlation between the jet power and the spin of the black holes. On the other hand, Russell et al. (2013); Fender \& Gallo (2014) did not find any such correlation in their study. Very recently, Aktar et al. (2015) calculated the mass loss from inviscid advective disc considering accretionejection model and found a feeble correlation between the maximum outflow rates and the spin of the black holes. This result possibly indicates that the existence of jet-spin correlation in the accretion-ejection system seems to be elusive.

\footnotetext{
* E-mail: ramiz@iitg.ernet.in (RA); sbdas@iitg.ernet.in (SD); anuj@isac.gov.in (AN); sreehari@physics.iisc.ernet.in (HS)
} 
Ramiz Aktar, Santabrata Das, Anuj Nandi, H. Sreehari

Extensive efforts were made to infer the exact mechanism of jet generation and the powering of jets (Nandi et al. 2001, Falcke et al. 2004 Fender et al. 2004, 2009, Steiner et al. 2013 Russell et al. 2013 McClintock et al. 2014 |Fender \& Gallo 2014). Several attempts were pursued to investigate the accretion-ejection coupling mechanism considering the inflowing matter to be sub-Keplerian in nature. In these works, both the accretion and ejection processes are overall governed by the conservation laws of gas dynamics, namely the conservation of mass, momentum and energy. In reality, during the course of the accretion process, subsonic rotating flow starts it journey from the outer edge of the disc with negligible radial velocity. Due to the influence of strong gravity, subsonic matter gradually gains its radial velocity while moving towards the black hole and becomes super sonic after crossing the sonic point. Depending on the angular momentum, accretion flow may contain multiple sonic points and in this case, accreting matter after passing through the outer sonic point experiences centrifugal barrier while moving further towards the horizon. This causes the piling of matter which eventually may trigger the shock transition. The feasibility of shock solutions and its implications have been studied extensively around non-rotating black holes (Fukue 1987 Chakrabarti | 1989, Lu et al. 1999, Becker \& Kazanas |2001. Das et al. 2001a Fukumura \& Tsuruta 2004, Chakrabarti \& Das 2004 Das 2007|. Das et al. 2009 |Sarkar \& Das 2016) as well as rotating black holes (Chakrabarti 1996b||Mondal \& Chakrabarti 2006, Das \& Chakrabarti 2008, Das et al. 2010 Aktar et al. 2015).

The advantage of the shock induced accretion solution is that due to shock compression, the post shock region of the accretion disc around black holes becomes hot and dense and eventually, innermost region of the disc does not remain confined around the disc equatorial plane, instead it becomes puffed up. Apparently, the post shock flow around the black holes behaves like a Comptonizing cloud (equivalently Post Shock Corona, hereafter PSC). During accretion, inflowing matter experiences excess thermal gradient force at PSC which drives a part of the infalling matter in the vertical direction to produce bipolar outflows. This underlying mechanism possibly establishes the coupling of outflow generation with the accretion dynamics. Extensive numerical simulations in hydrodynamics (HD) as well as magnetohydrodynamics (MHD) environment confirm the disk-jet connection where the generation of mass outflow takes place from the inner part of the disc (Molteni et al. 1994, 1996a. Machida et al. |2000 Koide et al.| 2002| McKinney \& Gammie 2004; De Villiers et al.|2005; Giri et al. |2010; Okuda 2014 Das et al. 2014, Okuda \& Das 2015)

Considering the above appealing mechanism of outflow formation, Chakrabarti (1999) and Das et al. (2001a) estimated the rate of mass loss from the disc self-consistently for adiabatic flow. Subsequently, the studies of outflow rates from accretion flows are demonstrated with variety and complexity of disc-jet structures (Das et al. 2001a Chattopadhyay \& Das 2007, Das \& Chattopadhyay 2008 $\mid \mathrm{Ku}-$ mar \& Chattopadhyay 2013 Aktar et al. 2015 Chattopadhyay \& Kumar 2016). Das \& Chattopadhyay (2008) performed a detail study of mass loss for dissipative steady accretion flows around non-rotating black holes including the effects of viscosity and Synchrotron cooling processes. Recently, Chattopadhyay \& Kumar (2016) further examined accretion-ejection solutions around Schwarzschild black hole in full general relativity. Meanwhile, Aktar et al. (2015) investigated the outflow properties around the rotating black holes for inviscid flow. In these studies, the effects of dissipative processes and the black hole rotation on the accretionejection solutions are carried out separately. It is therefore pertinent to investigate how the properties of the accretion as well as the outflow solutions depend on the black hole spin and the dissipation parameters which is apparently the main purpose of this work.

Accordingly, in this paper, we undertake a steady, axisymmetric, sub-Keplerian, viscous advective accretion flow around a rotating black hole. Indeed, as Chakrabarti (1996c) pointed out that the sub-Keplerian accretion around central black hole seems to be viable, when matter is accreted from the winds of the surrounding stars having very little angular momentum. Moreover, following the work of Narayan \& Yi (1994), here we introduce the effect of cooling in a parametric way. To avoid the complexities of full general relativistic calculations, we adopt the pseudo-Kerr potential (Mondal \& Chakrabarti 2006) that satisfactorily describes the spacetime geometry around the rotating black holes having spin parameter $a_{k} \lesssim 0.8$. In this problem, the bipolar jets and outflows are assumed to be launched from the PSC and the jets are assumed to be confined within the physically motivated jet geometry which is constructed based on the treatment proposed by Molteni et al. (1996a). We calculate the shock induced global transonic accretion solutions in presence and absence of thermally driven outflows and identify the parameter spaces spanned by the local energy $\left(\mathcal{E}_{\text {in }}\right)$ and angular momentum $\left(\lambda_{\text {in }}\right)$ at the inner sonic point $\left(x_{\text {in }}\right)$ of the inflowing mater in terms of the black hole spin. In addition, we also performed the classification of the parameter spaces as function of the viscosity and cooling parameters. We find that the available parameter space for shock is always reduced when outflow is present. It is observed that the accretion-ejection solutions exist for a wide range of the inflow parameters. Furthermore, we compute the maximum mass outflow rate $\left(R_{\dot{m}}^{\max }\right)$ in terms of viscosity parameter $(\alpha)$ by varying the inflow parameters and observed that $R_{\dot{m}}^{\max }$ is predominantly obtained for higher $\mathcal{E}_{\text {in }}$ and lower $\lambda_{\text {in }}$ values irrespective to the spin of the black holes. Next, we calculate the maximum values of the viscosity parameter $\left(\alpha^{\max }\right)$ as function of black hole spin $\left(a_{k}\right)$ both by excluding outflow $\left(\alpha_{\text {no }}^{\max }\right)$ and including outflows $\left(\alpha_{\mathrm{o}}^{\max }\right)$. Since the shock parameter space is shrunk when outflow is included, for a given $a_{k}$, we obtain $\alpha_{\mathrm{o}}^{\max }<\alpha_{\text {no }}^{\max }$ all throughout. Moreover, in our present analysis, we find that the estimated limits of $\alpha_{\mathrm{no}}^{\max } \sim 0.35$ and $\alpha_{\mathrm{o}}^{\max } \sim 0.25$ for non-rotating black hole $\left(a_{k}=0\right)$ are very much consistent with the results reported by Chakrabarti \& Das (2004); Kumar \& Chattopadhyay (2013). Following our present inflow-outflow formalism, we attempt to find out the plausible origin of the high frequency QPOs and also explore the possibility of constraining the spin of the black hole sources. As a representative case, we analyze the $R X T E^{1}$ satellite archival data of a particular observation of the black hole source GRO J1655-40 as it exhibits the highest observed QPO frequency ( $\sim 450 \mathrm{~Hz})$ among all the known black hole sources. Based

\footnotetext{
1 https://heasarc.gsfc.nasa.gov/docs/xte/xtegof.html
} 
on the present approach, we argue that the spin of GRO J1655-40 seems to be $a_{k} \geqslant 0.57$.

The remainder of the paper is organized as follows. In $\S 2$, we describe the governing equations for accretion and outflows. In the next section (§3), we discuss the solution methodology to calculate the inflow-outflow solutions in terms of the inflow parameters and estimate the mass outflow rates. In $\S 4$, we present the results in detail and discuss the possible observational implications of our formalism to constrain the spin of the black hole sources. Finally, we review our main conclusions in $\S 5$.

\section{MODEL EQUATIONS AND ASSUMPTIONS}

In this article, we consider a disc-jet system around a rotating black hole where we assume that the accretion disc lies along the equatorial plane and the jet geometry is considered in the off-equatorial plane about the black hole rotation axis (Molteni et al. 1996a Aktar et al. 2015, see Fig. 1). To represent the flow variables, we use the geometric unit system as $G=M_{\mathrm{BH}}=c=1$, where, $G, M_{\mathrm{BH}}$ and $c$ are the Gravitational constant, mass of the black hole and speed of light, respectively.

\subsection{Governing Equations for Accretion}

We consider a steady, geometrically thin, axisymmetric, viscous accretion flow around a rotating black hole. To avoid the complexity of full general relativistic calculation, we adopt the pseudo-Kerr potential approach to mimic the space-time geometry around a rotating black hole. The equations of motion for accreting matter are given by,

(i) The radial momentum equation:

$$
v \frac{d v}{d x}+\frac{1}{\rho} \frac{d P}{d x}+\frac{d \Phi_{e f f}}{d x}=0
$$

where, $v, P$ and $\rho$ represent the radial velocity, gas pressure and density of the accretion flow respectively. Here, $\Phi_{\text {eff }}$ denotes the pseudo-Kerr effective potential proposed by Chakrabarti \& Mondal (2006). The expression of $\Phi_{\text {eff }}$ is given by,

$$
\Phi_{\text {eff }}=-\frac{\mathcal{B}+\sqrt{\mathcal{B}^{2}-4 \mathcal{A C}}}{2 \mathcal{A}}
$$

where,

$$
\begin{aligned}
& \mathcal{A}=\frac{\epsilon^{2} \lambda^{2}}{2 x^{2}}, \\
& \mathcal{B}=-1+\frac{\epsilon^{2} \omega \lambda r^{2}}{x^{2}}+\frac{2 a_{k} \lambda}{r^{2} x} \\
& \mathcal{C}=1-\frac{1}{r-x_{0}}+\frac{2 a_{k} \omega}{x}+\frac{\epsilon^{2} \omega^{2} r^{4}}{2 x^{2}} .
\end{aligned}
$$

Here, $x$ and $r$ represent the cylindrical and spherical radial distance considering the black hole is located at the origin of the coordinate system and $\lambda$ is the specific angular momentum of the flow. Here, $x_{0}=0.04+0.97 a_{k}+0.085 a_{k}^{2}, \omega=$ $2 a_{k} /\left(x^{3}+a_{k}^{2} x+2 a_{k}^{2}\right)$ and $\epsilon^{2}=\left(x^{2}-2 x+a_{k}^{2}\right) /\left(x^{2}+a_{k}^{2}+2 a_{k}^{2} / x\right)$, $\epsilon$ is the redshift factor and $a_{k}$ represents the black hole rotation parameter defined as the specific spin angular momentum of the black hole. According to Chakrabarti \& Mondal
(2006), the above potential mimic the Kerr geometry quite satisfactorily for $a_{k} \leqslant 0.8$.

(ii) The mass conservation equation:

$$
\dot{M}=4 \pi \rho v x h
$$

where, $\dot{M}$ denotes the mass accretion rate which is constant everywhere except the region of mass loss and $4 \pi$ is the geometric constant. Considering the hydrostatic equilibrium in the vertical direction, the half-thickness of the disc $h(x)$ is obtained as,

$$
h(x)=a \sqrt{\frac{x}{\gamma \Phi_{r}^{\prime}}},
$$

where, $a$ is the adiabatic sound speed defined as $a=\sqrt{\frac{\gamma P}{\rho}}$ and $\gamma$ is the adiabatic index. Here, $\Phi_{r}^{\prime}=\left(\frac{\partial \Phi_{e f f}}{\partial r}\right)_{z<<x}$, and $z$ is the vertical height in the cylindrical coordinate system where $r=\sqrt{x^{2}+z^{2}}$ (Das et al. 2010).

(iii) The angular momentum distribution equation:

$$
v \frac{d \lambda}{d x}+\frac{1}{\Sigma x} \frac{d}{d x}\left(x^{2} W_{x \phi}\right)=0 .
$$

Here, we assume that the viscous stress is dominated by the $x \phi$ component and is denoted by $W_{x \phi}$. In this work, for the sake of completeness, we consider the viscous stress which is given by Chakrabarti (1996a),

$$
W_{x \phi}^{(1)}=-\alpha\left(W+\Sigma v^{2}\right),
$$

where, $W=2 I_{n+1} P h(x)$ and $\Sigma=2 I_{n} \rho h(x)$ are the vertically integrated pressure and density, respectively (Matsumoto et al. 1984) and $\alpha$ refers to the viscosity parameter. When radial velocity of the inflowing matter is insignificant as in the case of Keplerian disc, equation (6) reduces to the seminal viscosity prescription of Shakura \& Sunyaev (1973). It is to be noted that an alternative expression of the viscous shear stress is also considered while modeling the accretion flow around black hole (Chakrabarti 1996a, and reference therein) which is given by,

$$
W_{x \phi}^{(2)}=\eta x \frac{d \Omega}{d x}
$$

where, $\Omega(x)$ is the angular velocity of the accreting matter. And finally,

(iv) The entropy generation equation:

$$
\Sigma v T \frac{d s}{d x}=Q^{+}-Q^{-}=f Q^{+} ; f=1-\frac{Q^{-}}{Q^{+}},
$$

where, $T$, and $s$ are the temperature and entropy density of the accretion flow, respectively. Here, $Q^{+}$and $Q^{-}$denote the heat gain and lost by the flow. Here, we introduce a parametric cooling factor $f$ in our calculation following the work of Narayan \& Yi (1994), where the value of $f$ lies in the range $0 \leqslant f \leqslant 1$. When $f=1$, accretion flow behaves like advection dominated whereas for $f=0$, flow becomes cooling dominated.

After some simple algebra, equation (8) yields as,

$$
\frac{v}{\gamma-1}\left[\frac{1}{\rho} \frac{d P}{d x}-\frac{\gamma P}{\rho^{2}} \frac{d \rho}{d x}\right]=-\frac{f Q^{+}}{\rho h}=-H .
$$

where, we define $H=\frac{f Q^{+}}{\rho h}$. Due to viscous shear, accreting matter is heated up and we compute the heating of the flow 


\section{Ramiz Aktar, Santabrata Das, Anuj Nandi, H. Sreehari}

as $Q^{+}=W_{x \phi}^{2} / \eta$ where $\eta$ represents the dynamical viscosity coefficient. To calculate $Q^{+}$, when $W_{x \phi}^{(1)}$ is used, the contribution of viscous shear is being sacrificed. On the other hand, when $W_{x \phi}^{(2)}$ is used, second order derivative of $\Omega$ appeared in the sonic point analysis and equations become difficult to solve. To avoid such limitations, however, retaining the memory of $d \Omega / d x$ intact, we adopt the mixed shear stress prescription (Chakrabarti 1996a) where the combination of both shear stresses are considered. In the present analysis, therefore the heating term $Q^{+}$yields as,

$$
Q^{+}=\frac{W_{x \phi}^{(1)} W_{x \phi}^{(2)}}{\eta}=-\alpha\left(W+\Sigma v^{2}\right)\left(x \frac{d \Omega}{d x}\right) .
$$

Using equation (10), we obtain the simplified expression of $H$ as,

$$
H=\frac{f Q^{+}}{\rho h}=f A\left(g a^{2}+\gamma v^{2}\right)\left(x \frac{d \Omega}{d x}\right),
$$

where, $A=-2 \alpha I_{n} / \gamma$ and $g=I_{n+1} / I_{n}$. Here, $n=(\gamma-1)^{-1}$ is the polytropic index. $I_{n}$ and $I_{n+1}$ are the constant factors arising while carrying out the vertical integration of density and pressure at a given radial coordinate $x$ (see Matsumoto et al. (1984)).

\subsection{Sonic point conditions}

In the accretion process around black hole, radial velocity of the inflowing matter at the outer edge remains negligibly small although matter enters into the black hole with radial velocity equal to the speed of light. Therefore, the velocity of the inflowing matter is likely to match with the sound speed at some point where flow changes its sonic character from subsonic to supersonic state. Such a point is called as sonic point. Depending on the initial parameters, flow may contain more than one sonic points. Flow containing multiple sonic points is of our interest as it may possess stationary shock when the shock conditions are satisfied. In order to investigate the properties of the shock wave and its implication for black hole accretion, study of sonic point properties is therefore essential. Accordingly, we carry out sonic point analysis following Chakrabarti (1989) and obtain the first order linear differential equation using equations (1), (3), (5) and (9) as,

$$
\frac{d v}{d x}=\frac{N}{D}
$$

where,

$$
\begin{aligned}
N= & -\frac{f A \alpha\left(g a^{2}+\gamma v^{2}\right)^{2}}{\gamma v x}-\frac{3 a^{2} v}{(\gamma-1) x}+\frac{a^{2} v}{(\gamma-1)}\left(\frac{d \ln \Phi_{r}^{\prime}}{d x}\right) \\
& +\left[\frac{2 f A \alpha g\left(g a^{2}+\gamma v^{2}\right)}{v}+\frac{(\gamma+1) v}{(\gamma-1)}\right]\left(\frac{d \Phi_{e f f}}{d x}\right) \\
& -\frac{3 f A \alpha g a^{2}\left(g a^{2}+\gamma v^{2}\right)}{\gamma v x}+\frac{f A \alpha g a^{2}\left(g a^{2}+\gamma v^{2}\right)}{\gamma v}\left(\frac{d \ln \Phi_{r}^{\prime}}{d x}\right) \\
& +\frac{2 f A \lambda\left(g a^{2}+\gamma v^{2}\right)}{x^{2}},
\end{aligned}
$$

and

$$
D=\frac{2 a^{2}}{(\gamma-1)}-\frac{(\gamma+1) v^{2}}{(\gamma-1)}-f A \alpha\left(g a^{2}+\gamma v^{2}\right)\left[(2 g-1)-\frac{g a^{2}}{\gamma v^{2}}\right] .
$$

We calculate the differential form of angular momentum using the expression of equation (5) as,

$\frac{d \lambda}{d x}=\frac{\alpha}{\gamma v}\left(g a^{2}+\gamma v^{2}\right)+\frac{2 \alpha x g a}{\gamma v}\left(\frac{d a}{d x}\right)+\alpha x\left(1-\frac{g a^{2}}{\gamma v^{2}}\right)\left(\frac{d v}{d x}\right)$,

and also we calculate the gradient of sound speed using equations (1-4) as,

$$
\frac{d a}{d x}=\left(\frac{a}{v}-\frac{\gamma v}{a}\right) \frac{d v}{d x}+\frac{3 a}{2 x}-\frac{a}{2}\left(\frac{d \ln \Phi_{r}^{\prime}}{d x}\right)-\frac{\gamma}{a}\left(\frac{d \Phi_{e f f}}{d x}\right) .
$$

The inner boundary condition of black hole demands that the flow should be smooth everywhere between the outer edge and the horizon. Therefore, the radial velocity gradient must be finite everywhere. To maintain this, at the sonic point, both numerator $N$ and denominator $D$ of Eq. (12) must vanish simultaneously (Chakrabarti 1989). Setting $D=0$, we obtain the Mach number $(M=v / a)$ expression at the sonic point as,

$$
M_{c}^{2}=\frac{-m_{b}-\sqrt{m_{b}^{2}-4 m_{a} m_{c}}}{2 m_{a}},
$$

where,

$$
\begin{aligned}
& m_{a}=-f A \alpha \gamma^{2}(\gamma-1)(2 g-1)-\gamma(\gamma+1), \\
& m_{b}=2 \gamma-2 f A \alpha g \gamma(\gamma-1)(g-1), \\
& m_{c}=f A \alpha g^{2}(\gamma-1) .
\end{aligned}
$$

The sound speed at the sonic point is found out by setting numerator $N=0$ and is given by,

$$
a\left(x_{c}\right)=\frac{-a_{2}-\sqrt{a_{2}^{2}-4 a_{1} a_{3}}}{2 a_{1}}
$$

where,

$$
\begin{aligned}
a_{1}= & -\frac{f A \alpha\left(g+\gamma M_{c}^{2}\right)^{2}}{\gamma x}-\frac{3 M_{c}^{2}}{(\gamma-1) x}+\frac{M_{c}^{2}}{(\gamma-1)}\left(\frac{d \ln \Phi_{r}^{\prime}}{d x}\right) \\
& -\frac{3 f A \alpha g\left(g+\gamma M_{c}^{2}\right)}{\gamma x}+\frac{f A \alpha g\left(g+\gamma M_{c}^{2}\right)}{\gamma}\left(\frac{d \ln \Phi_{r}^{\prime}}{d x}\right), \\
a_{2}= & \frac{2 f A \lambda M_{c}\left(g+\gamma M_{c}^{2}\right)}{x^{2}}, \\
a_{3}= & {[2 f A \alpha g(g+\gamma)}
\end{aligned}
$$

Here, $M_{c}$ represents the Mach number at the sonic point.

\subsection{Equations for Outflow}

In this work, we consider that the accretion flow geometry is resided around the equatorial plane and the jet or outflow geometry is described in the off-equatorial plane about the axis of rotation of the black hole (Molteni et al. 1996a: Chattopadhyay \& Das 2007). As the jets are tenuous in nature, the differential rotation of the outflowing matter is expected to be negligibly small and thus, we ignore the viscosity while describing outflow. Further, the outflowing matter is considered to obey the polytropic equation of state as $P_{j}=K_{j} \rho_{j}^{\gamma}$, where, the suffix ' $j$ ' denotes the outflow variables and $K_{j}$ 


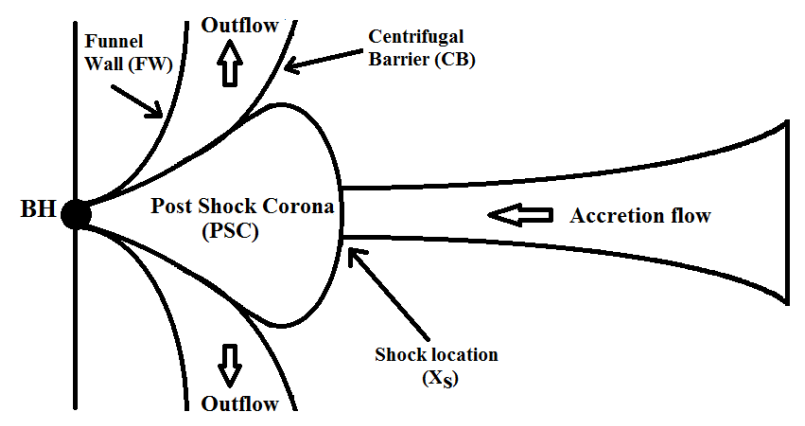

Figure 1. Schematic diagram of disc-jet geometry is shown. Accretion flow, post-shock corona (PSC) and outflow are depicted in the figure. Also, we show the shock location $\left(x_{s}\right)$, centrifugal barrier (CB) and funnel wall (FW). Here, the central black hole is indicated as BH. See text for details.

denotes the measure of specific entropy of the jet. Subsequently, the equations of motion of the outflowing matter are given by,

(i) The energy conservation equation of outflow:

$$
\mathcal{E}_{j}=\frac{1}{2} v_{j}^{2}+\frac{a_{j}^{2}}{\gamma-1}+\Phi_{e f f},
$$

where, $\mathcal{E}_{j}$ represents the specific energy of the outflow, $v_{j}$ is the outflow velocity and $a_{j}$ is the sound speed of the outflow, respectively.

(ii) the mass conservation equation of outflow:

$$
\dot{M}_{\text {out }}=\rho_{j} v_{j} \mathcal{A}_{j}
$$

where, $\dot{M}_{\text {out }}$ represents the outflowing mass rate and $\mathcal{A}_{j}$ refers to the area function of the jet. We estimate $\mathcal{A}_{j}$ by using the radius of two boundary surfaces, namely the centrifugal barrier (CB) and the funnel wall (FW) (Molteni et al. 1996a) (see Fig. 1). The centrifugal barrier (CB) is obtained by defining the pressure maxima surface as $\left(d \Phi^{e f f} / d x\right)_{r_{C B}}=0$ and the funnel wall (FW) stands for the pressure minimum surface which is defined by the null effective potential as $\left.\Phi^{e f f}\right|_{r_{F W}}=0$ (Molteni et al. 1996a). In general, the jet streamlines within the jet geometry are not exactly parallel to the jet area vector and therefore, in order to calculate the jet area function, we introduce a geometric factor corresponding to the projection of the jet streamlines on the jet cross-section. Accordingly, the jet area function is obtained as (Kumar \& Chattopadhyay 2013),

$$
\mathcal{A}_{j}=\frac{2 \pi\left(x_{C B}^{2}-x_{F W}^{2}\right)}{\sqrt{1+\left(d x_{j} / d y_{j}\right)^{2}}},
$$

where, $\sqrt{1+\left(d x_{j} / d y_{j}\right)^{2}}$ is projection factor of the jet area. Here, $x_{C B}$ and $x_{F W}$ refer to the radius of the centrifugal barrier and funnel wall in the cylindrical coordinate system, respectively. The corresponding spherical radius of the jet is given by $r_{j}=\sqrt{x_{j}^{2}+y_{j}^{2}}$. With this consideration, we carry out the sonic point analysis for outflows following Das \& Chattopadhyay (2008). Subsequently, we use the jet sonic point properties while obtaining the outflow solution.

\section{SOLUTION METHODOLOGY}

In the accretion process, rotating matter experiences centrifugal repulsion while falling inward that eventually causes a virtual barrier around the black hole. Depending on the initial parameters, such a centrifugal barrier can trigger discontinuous transition in the flow variables in the form of shock. Because of shock compression, since the post-shock flow (i.e., PSC) becomes hot and dense, excess thermal gradient force at PSC is developed that deflects a part of the infalling matter along the black hole rotation axis in the form of outflows/jets. In the present analysis, we consider the outflowing matter to be emerged out between the centrifugal barrier (CB) and funnel wall (FW) surfaces. Following the above consideration, we move forward to obtain the self-consistent accretion-ejection solutions that are coupled via Rankine-Hugoniot (hereafter $\mathrm{RH}$ ) shock conditions. In the presence of outflow, the RH conditions are given by,

(i) the conservation of energy flux:

$$
\mathcal{E}_{+}=\mathcal{E}_{-},
$$

where, the quantities with subscripts ' - ' and ' + ' sign indicate the value of the variables before and after the shock, respectively. Here, $\mathcal{E}$ refers to the local energy of the flow and is obtained from equation (1) as $\mathcal{E}(x)=v^{2}(x) / 2+a^{2}(x) /(\gamma-$ 1) $+\Phi_{\text {eff }}(x)$.

(ii) the conservation of mass flux:

$$
\dot{M}_{+}=\dot{M}_{-}-\dot{M}_{\text {out }}=\dot{M}_{-}\left(1-R_{\dot{m}}\right) .
$$

It is already pointed out that during mass loss, a part of the inflowing matter is emerged out as outflow while the rest is advected in to the black hole. Here, we denote pre-shock and post-shock accretion rate as $\dot{M}_{-}$and $\dot{M}_{+}$, respectively and define the outflow rate as $R_{\dot{m}}=\dot{M}_{\text {out }} / \dot{M}_{-}$.

And, finally we have,

(iii) the conservation of momentum flux:

$$
W_{+}+\Sigma_{+} v_{+}^{2}=W_{-}+\Sigma_{-} v_{-}^{2} .
$$

where, $W$ and $\Sigma$ stands for the vertically integrated pressure and density stated before (Das et al. 2001b).

In our accretion-ejection model, outflow is considered to be originated from the post shock region and accordingly, we assume that the outflow is essentially launched with the same density as in the PSC i.e., $\rho_{j}=\rho_{+}$. Using equations $(3),(20)$ and $(22 \mathrm{~b})$, the mass outflow rate is then computed as,

$$
R_{\dot{m}}=\frac{\dot{M}_{\text {out }}}{\dot{M}_{-}}=\frac{R v_{j}\left(x_{s}\right) \mathcal{A}_{j}\left(x_{s}\right)}{4 \pi \sqrt{\frac{1}{\gamma}} x_{s}^{3 / 2} \Phi_{r}^{\prime-1 / 2} a_{+} v_{-}},
$$

where, $R$ is the compression ratio defined as $R=\Sigma_{+} / \Sigma_{-}$. In addition, $v_{j}\left(x_{s}\right)$ and $\mathcal{A}_{j}\left(x_{s}\right)$ are the jet velocity and the jet area function calculated at the shock, respectively. Here, we employ the successive iteration method to calculate $R_{\dot{m}}$ self-consistently. In this method, initially we start with $R_{\dot{m}}=0$. Using $\mathrm{RH}$ shock conditions, we calculate the virtual shock location $x_{s}^{v}$ for a given set of inflow variables, namely $\left(\mathcal{E}_{i n j}, \lambda_{i n j}, a_{k}, \alpha, f\right)$. Subsequently, we use $\mathcal{E}_{j}=\mathcal{E}_{+}$ and $\lambda_{j}=\lambda_{+}$to calculate the jet sonic point using equations (19) and (20). Starting form the jet sonic point, we integrate the jet equations towards the black hole horizon in order to calculate the jet variables and continue to do so up to the jet base which is equivalently the shock location. Utilizing 
Ramiz Aktar, Santabrata Das, Anuj Nandi, H. Sreehari

these jet variables, we compute the virtual $R_{\dot{m}}^{v}$ which we use further in the $\mathrm{RH}$ shock conditions to get a new shock location. We continue the iteration process until the shock location converges to the actual location at $x_{s}$ and finally obtain $R_{\dot{m}}$ corresponding to $x_{s}$.

\section{RESULTS AND DISCUSSIONS}

In this section, we present our model solutions for various sets of initial parameters of the accretion flow. In particular, here we depict the effect of dissipation processes, namely viscosity and cooling on the mass loss from the accretion disc around rotating black hole. In the first section, we investigate the effect of viscosity on the outflow rates in the advection-dominated regime (i.e., $f=1$ ) and in the next section, we show the effect of cooling on outflow rates in the cooling-dominated regime (i.e., $f \rightarrow 0$ ). As mentioned in $\S 2$, the pseudo-Kerr potential Chakrabarti \& Mondal 2006) adopted in this work describes the general relativistic features of space-time geometry quite satisfactorily in the range $-1 \leqslant a_{k} \leqslant 0.8$. However, for the sake of completeness, here we present results beyond $a_{k}>0.8$ to examine the overall characteristics of the accretion solution for the maximally rotating black hole. Though it introduces error $\sim 20 \%$ as far as marginally stable orbit is concerned for the maximally rotating case (Chakrabarti \& Mondal 2006), we argue that the overall findings of the accretion solutions would not deviate severely. In addition, it is known that the adiabatic index $(\gamma)$ depends on the ratio of the thermal energy to the rest energy of the flow and the theoretical limit of $\gamma$ lies in the range $4 / 3 \leqslant \gamma \leqslant 5 / 3$ (Frank et al. 2002). In this work, for the purpose of representation, we consider $\gamma=1.4$ all throughout unless otherwise stated.

\subsection{Effect of viscosity on outflow rates}

In Fig. 2, we present the global dissipative viscous accretion solutions around the rotating black holes for various sets of inflow parameters. All these solutions contain shock waves. Here, the flow parameters at the outer edge is chosen as $x_{\text {inj }}=500, \lambda_{\text {inj }}=2.656$ and $\mathcal{E}_{\text {inj }}=0.00135$, respectively and the black hole spin is considered as $a_{k}=0.76$. In Fig. 2a, we show the Mach number $(M=v / a)$ variation of the inflowing matter with the radial distance in absence of mass loss. The solid, dashed and dotted curves are for the viscosity parameter $\alpha=0.0$ (black), $\alpha=0.003$ (red) and $\alpha=0.0046$ (blue), respectively. We find that the shock front moves inward towards the black hole horizon with the increase of $\alpha$. This is simply because the increase of $\alpha$ enhances the angular momentum transport towards the outer edge of the disc that eventually instigates the debilitating of centrifugal barrier around the black hole. Effectively, this compels the shock front to move inward in order to preserve the pressure balance across it. In the figure, vertical arrows indicate the shock transition and the location of the shock are obtained as $x_{s}=42.03,22.85$ and 15.59 for inviscid case, $\alpha=0.0,0.003$ and 0.0046 , respectively. In Fig. 2b, we display the accretion solution in presence of mass loss for the same set of inflow parameters as in Fig. 2a. In the present model, the outflow is launched from the post-shock region. Therefore, when a part of the inflowing

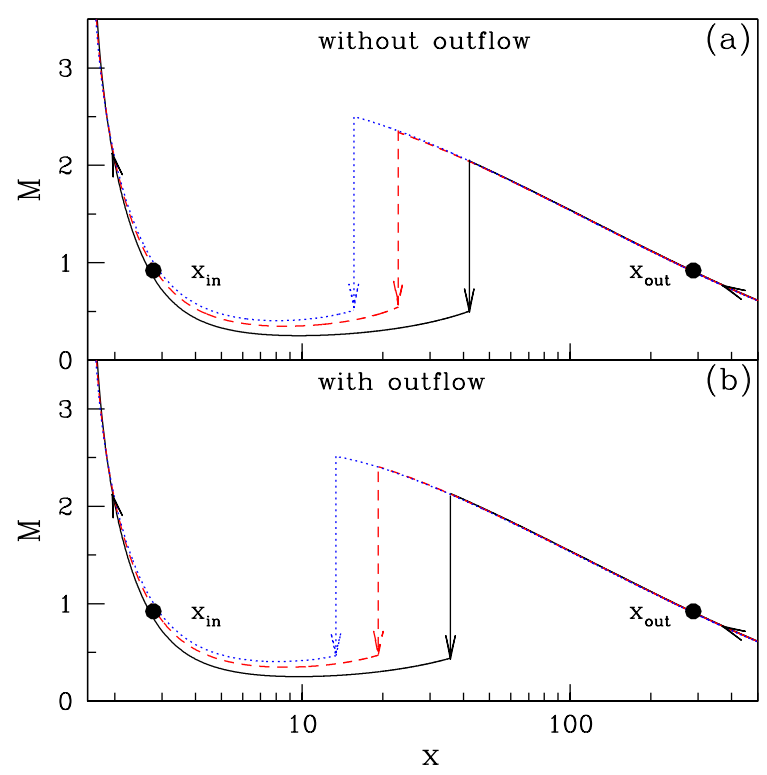

Figure 2. Variation of inflow Mach number $M=(v / a)$ with radial distance $(x)$. Solid, dashed and dotted curves are for $\alpha=0.0$ (black), 0.003 (red) and 0.0046 (blue), respectively. Upper panel is for without outflow and the lower panel is for with outflow. Here, we fix $a_{k}=0.76$. See text for details.

matter is emerged out from the disc as outflow, the effective pressure at the inner part of the disc is reduced and eventually the shock front is propelled towards the black hole even further in order to maintain the pressure balance across the shock. The solid, dashed and dotted curves denote the results corresponding to the viscosity parameters, shock locations and outflow rates as, $\left(\alpha, x_{s}, R_{\dot{m}}\right)=$ $(0.0,35.76,0.0346)$ (black), $(0.003,19.24,0.0220)$ (red) and $(0.0046,13.36,0.0182)$ (blue), respectively. In both the panels, filled circles indicate the inner $\left(x_{\text {in }}\right)$ and outer $\left(x_{\text {out }}\right)$ sonic points and arrows represent the flow direction.

In Fig. 3, we investigate the effect of viscosity parameter on the inflow-outflow solutions around the rotating black holes. Here, we fix the injection radius of the inflowing matter at $x_{\mathrm{inj}}=500$ and the corresponding inflow energy $\left(\mathcal{E}_{\text {inj }}\right)$ and angular momentum $\left(\lambda_{\text {inj }}\right)$ at $x_{\text {inj }}$ are $\left(\mathcal{E}_{\text {inj }}, \lambda_{\text {inj }}\right)=(0.00135,2.656)$, respectively. We vary the spin of the black hole $a_{k}$ from 0.78 to 0.7 from the right most (dot-big-dashed) to the left most curves (solid) with an interval $\Delta a_{k}=0.02$. In the upper panel (Fig. 3a), we show the variation of shock location with the viscosity parameter $\alpha$ in presence of mass loss. As it is seen in Fig. 2, here also we find that shock $\left(x_{s}\right)$ proceeds closer to the black hole with the increase of viscosity parameter $(\alpha)$. This clearly indicates that the effective size of the PSC decreases with the increase of $\alpha$. As a consequence, the fraction of inflowing matter intercepted by the PSC is reduced that eventually produce feeble outflow rate when $\alpha$ is increased. In Fig. 3b, we plot the outflow rates $\left(R_{\dot{m}}\right)$ corresponding to Fig. 3a. In Fig. 3c, we study the compression ratio $(R)$ of shocked accretion flow in presence of mass loss as function of $\alpha$. Compression ratio essentially measures the density compression across the shock and is defined as the ratio of post-shock density to the pre-shock density. As the shock moves closer 


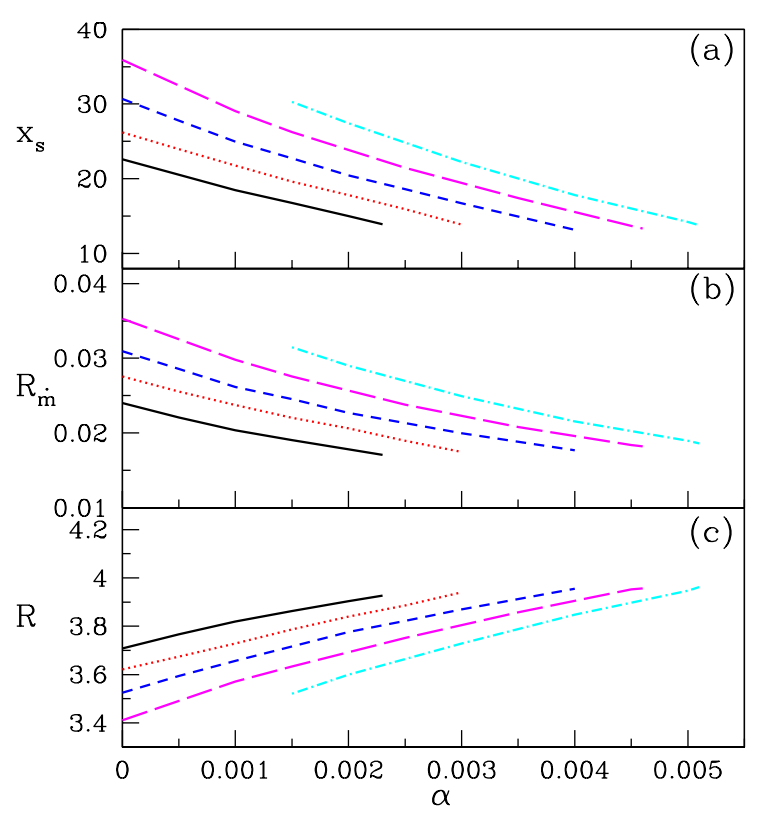

Figure 3. Variation of (a) shock location $\left(x_{s}\right)$, (b) outflow rate $\left(R_{\dot{m}}\right)$ and (c) compression ratio $(R)$ as a function of viscosity parameter $\alpha$ for $a_{k}=0.78$ to 0.70 , where $\Delta a_{k}=0.02$ (right to left). Here, we fix the outer boundary at $x_{\text {inj }}=500$. The angular momentum and energy of the flow at $x_{\text {inj }}$ is chosen as $\lambda_{\text {inj }}=2.656$ and $\mathcal{E}_{\text {inj }}=0.00135$, respectively. See text for further details.

to the horizon, flow experiences enhanced compression and therefore, $R$ increases with the increase of $\alpha$.

In Fig. 4, we show the effect of black hole rotation on the shock properties and the mass outflow rate. For this analysis, we choose the outer boundary of the disc at $x_{\mathrm{inj}}=300$ with specific energy and angular momentum as $\mathcal{E}_{\text {inj }}=0.0025$ and $\lambda_{\text {inj }}=2.80$, respectively. In the upper $(a)$, middle $(b)$ and bottom (c) panels of Fig. 4, we plot shock locations $\left(x_{s}\right)$, outflow rates $\left(R_{\dot{m}}\right)$ and compression ratio $(R)$ as function of black hole spin $\left(a_{k}\right)$, respectively. In all the panels, we vary the viscosity parameter from $\alpha=0.003$ to 0.007 with an interval $\Delta \alpha=0.001$ from left most to the right most curve. We observe that for fixed outer boundary condition, the shock location $\left(x_{s}\right)$ moves away from the black hole horizon with the increase of black hole rotation $a_{k}$ for flows with fixed $\alpha$. As a result, the effective area of PSC intercepted by the inflowing matter is increased and consequently, the amount of the inflowing matter deflected by the PSC is also increased yielding enhanced mass outflow rates $R_{\dot{m}}$. In contrary, we find that $R_{\dot{m}}$ decreases with the increase of $\alpha$ for fixed $a_{k}$ as seen in Fig. 3. In addition, as $x_{s}$ recedes away from the black hole horizon with the increase of $a_{k}$ for flows with constant $\alpha$, the compression ratio $(R)$ is also decreased as shown in Fig. 4c. On the other hand, for fixed $a_{k}$, as $\alpha$ increased, both $x_{s}$ and $R_{\dot{m}}$ decreases whereas $R$ increases. This findings are compatible with results of Aktar et al. (2015).

We proceed further to examine the region of parameter space that permits stationary shock solutions. As it is already pointed out (see Fig. 2-4) that the shock induced global accretion solutions are not the isolated solutions, instead the solutions of this kind can be obtained for a wide range of inflow parameters. Moreover, in our model, since the outflow is originated from the PSC, it is worthy to identify

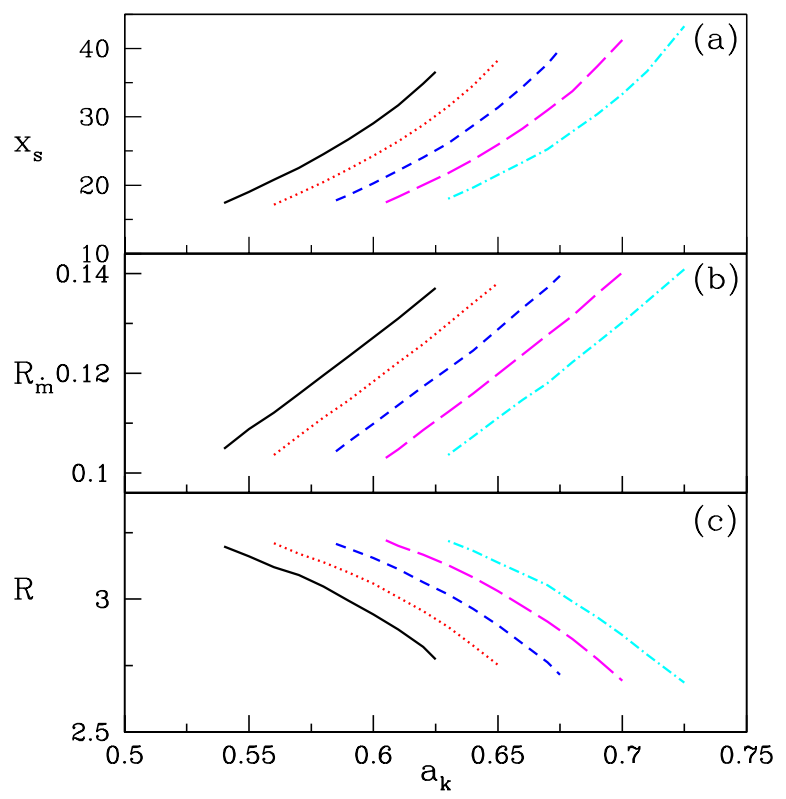

Figure 4. Plot of (a) shock location $\left(x_{s}\right)$, (b) outflow rate $\left(R_{\dot{m}}\right)$ and (c) compression ratio $(R)$ variation as function of spin $a_{k}$ for $\alpha=0.003$ to 0.007 , where $\Delta \alpha=0.001$ (left to right). Here, we fix the outer boundary at $x_{\mathrm{inj}}=300$ and choose $\lambda_{\mathrm{inj}}=2.80$ and $\mathcal{E}_{\mathrm{inj}}=0.0025$, respectively. See text for details.

the shock parameter space in terms of the viscosity parameter $(\alpha)$ and the spin of the black hole $\left(a_{k}\right)$. In Fig. 5 , we classify the region of the shock parameter space spanned by the angular momentum $\left(\lambda_{\text {in }}\right)$ and energy $\left(\mathcal{E}_{\text {in }}\right)$ of the flow measured at the inner sonic point $\left(x_{\mathrm{in}}\right)$. The results displayed in Fig. 5(a-c) are obtained for $a_{k}=0.0(a), 0.4(b)$ and 0.8 $(c)$, respectively. The corresponding viscosity parameters are marked in each panel. The solid curves separate the shock parameter space when mass loss from the disc is ignored whereas the dotted curves represent the shocked parameter space including mass loss. In presence of outflow, the effective area of the PSC becomes smaller (see Fig. 2) and therefore, the resulting parameter space is reduced in comparison to the no mass loss case, particularly in the lower angular momentum and higher energy side. When the input parameters are chosen from these parts of the parameter space ignoring outflow, accretion flow encounters shock transition very close to the black hole (Das et al. 2001a). After that when outflow is allowed to be launched from the PSC, shock ceases to exist. Subsequently, we study the role of viscosity in classifying the parameter space. In the accretion flow, the presence of viscosity manifests dual effects. Firstly, viscosity transports angular momentum outward reducing its value at the inner edge of the disc and secondly, flow is heated up due to the effect of viscous dissipation. Hence, as the viscosity is increased, the shock parameter space is shrunk from the both ends of the angular momentum range and also shifted towards the higher energy domain. Moreover, with the inclusion of viscosity, flow becomes dissipative and consequently, the possibility of standing shock formation is reduced (Chakrabarti \& Das 2004, Das 2007) and finally parameter space for standing shock disappears when the critical viscosity limit is reached. Interestingly, time varying shock solutions may still exist beyond the critical viscosity 
Ramiz Aktar, Santabrata Das, Anuj Nandi, H. Sreehari
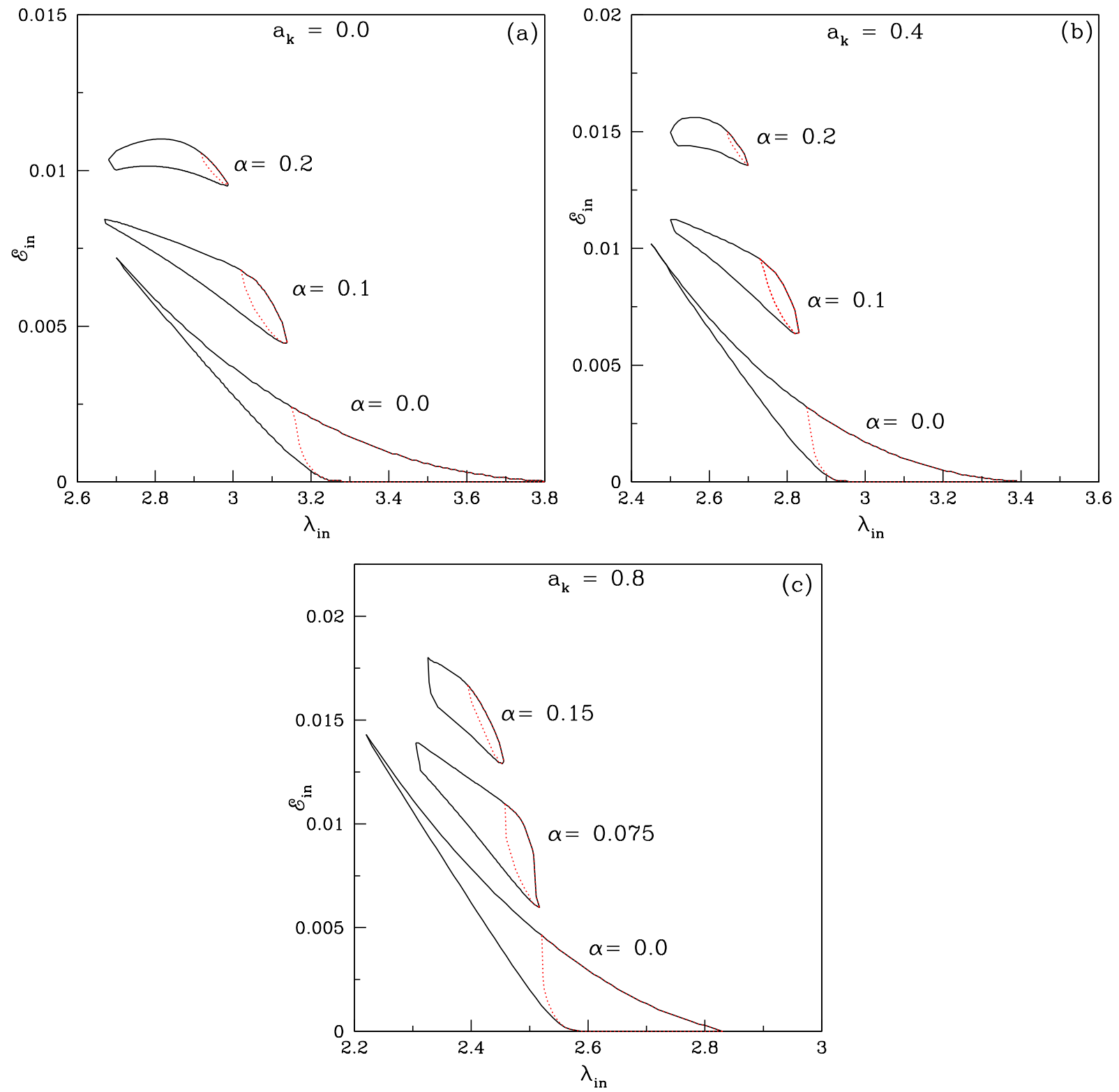

Figure 5. Identification of parameter space for shock in $\lambda_{\text {in }}-\mathcal{E}_{\text {in }}$ plane is shown as function of viscosity parameter $(\alpha)$ for various black hole spin parameter $\left(a_{k}\right)$. Solid curves denote the shock parameter space in absence of mass loss whereas dotted curves separate the shock parameter space in presence of mass loss. Fig. 5a, 5b and $5 \mathrm{c}$ illustrate the results for $a_{k}=0.0,0.4$ and 0.8 , respectively. See text for further details.

limit Chakrabarti \& Das 2004. Das et al. 2014). Numerical simulations already explored these oscillatory behavior of shock solutions (Lee et al. 2011, Das et al. 2014, Das \& Aktar 2015. Suková \& Jianuk 2015) which successfully describe the Quasi-periodic Oscillations (QPOs) and periodic mass loss phenomena observed in several astrophysical black hole systems (Chakrabarti et al. 2002, Nandi et al. 2012, Radhika et al. 2016a). A comprehensive study of time dependent global accretion solution around rotating black hole is considered as a future work as it is beyond the scope of the present paper and will be reported elsewhere.

We continue the study of shock properties in terms of the input flow parameters. Towards this, we investigate the maximum value of the viscosity parameters $\left(\alpha_{\text {no }}^{\max }\right.$ and $\left.\alpha_{\mathrm{o}}^{\max }\right)$ that allows steady shock solutions in absence $\left(R_{\dot{m}}=0\right)$ as well as in presence $\left(R_{\dot{m}} \neq 0\right)$ of mass loss. While doing so, we first fix the black hole spin $\left(a_{k}\right)$ and vary the remaining flow parameters freely. In Fig. 6 , we depict the variation of $\alpha^{\max }$ as function of $a_{k}$ where the upper panel is for without mass loss case and the lower panel is for with mass loss case. We already pointed out that the pseudo-Kerr potential adopted here describes the space time geometry around the black hole satisfactorily for $a_{k} \leqslant 0.8$. However, we present results for $a_{k}>0.8$ which are depicted with shaded region. Here, we argue that the obtained results demonstrate the overall findings of the accretion solutions at least qualitatively (Chakrabarti \& Mondal 2006). We find that the upper limit of viscosity parameter $\alpha^{\text {max }}$ for shock is anti-correlated with 


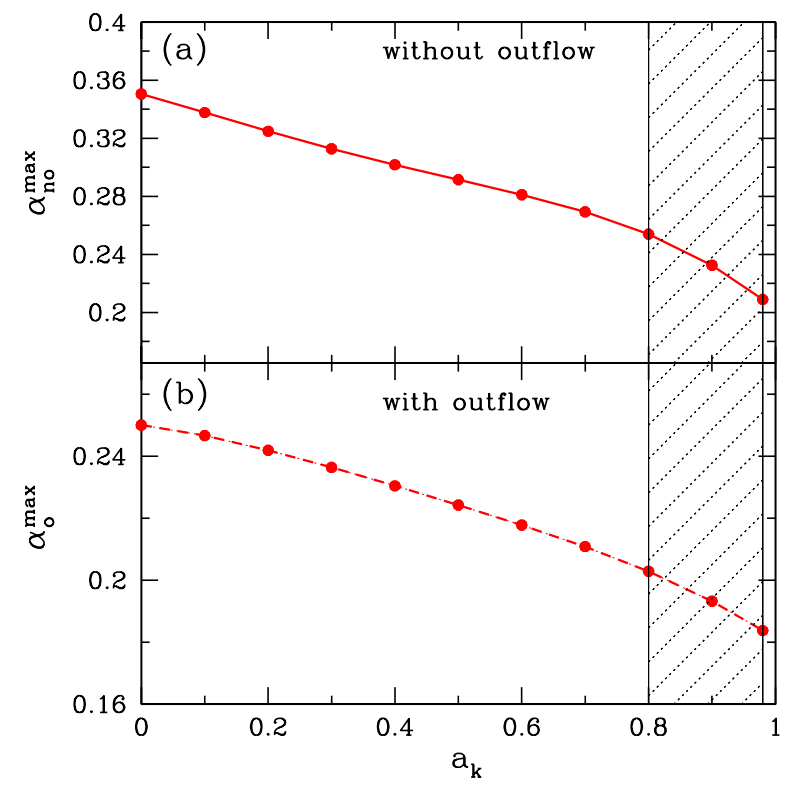

Figure 6. Variation of maximum viscosity parameter $\left(\alpha^{\max }\right)$ with spin of the black hole $a_{k}$ that allows standing shock. In the upper panel $(a)$, filled circles connected with the solid curve denotes $\alpha_{\text {no }}^{\max }$ in absence of outflow and in the lower panel $(b)$, filled circles joined with the dashed curve represents $\alpha_{\mathrm{o}}^{\max }$ in presence of outflow. In both the panels, results within the shaded region are obtained for $a_{k}>0.8$. See text for further details.

the spin $a_{k}$ in both the panels. In general, at the lower viscosity parameter, the sub-Keplerian flow joins with the Keplerian disc far away from the black hole horizon. This eventually increases the possibility of possessing multiple sonic points including shock waves. On the other hand, at the high viscosity limit, (i.e., $\alpha>\alpha^{\max }$ ), Keplerian disc comes very close to the black hole horizon allowing the flow to pass only through the single sonic point (Chakrabarti 1996a). In addition, the rotation of the black hole drags the accreting matter towards the horizon by its strong gravitational pull. Therefore, the possibility of the formation of steady shock in the higher viscosity domain for increasing $a_{k}$ becomes rarer. It is to be noted that the obtained $\alpha^{\max }$ for non-rotating black hole is consistent with the results of Chakrabarti \& Das (2004). When outflow is considered, the possibility of shock formation is decreased (see Fig. 5) and therefore, the obtained $\alpha^{\max }$ for $R_{\dot{m}} \neq 0$ becomes smaller compared to case with $R_{\dot{m}}=0$ (Kumar \& Chattopadhyay 2013).

In Fig. 7, we present the outflow parameter spaces for various $\alpha$ where each panel demonstrates the two dimensional surface projection of the three dimensional plot spanned with $\mathcal{E}_{\text {in }}, \lambda_{\text {in }}$ and $R_{\dot{m}}$, respectively. Here, we choose $a_{k}=0.5$ and vary the viscosity parameter as $\alpha=0.0$ (topleft), 0.05 (top-right), 0.1 (bottom-left) and 0.15 (bottomright). In each panel, the color-coded bar represents the range of $R_{\dot{m}}$ which is obtained from our model calculation. Moreover, the color coded contours are drawn with an interval of $\Delta R_{\dot{m}}=0.02$ starting from $R_{\dot{m}}^{\max }$ up to its minimum value. In other words, the different colors embedded with color coded contours illustrate the overall span of $2 \%$ outflow rate altogether. In addition, we find that the effective bounded region of the outflow parameter space gradually shrinks with the increase of the viscosity parameter $\alpha$ as is seen in Fig. 5. It is to be noted that in order to obtain the maximum outflow rate $\left(R_{\dot{m}}^{\max }\right)$, the inflow parameters must lie in the higher $\mathcal{E}_{\text {in }}$ and lower $\lambda_{\text {in }}$ domains irrespective to the value of the viscosity parameter $\alpha$.

In the course of our study, we put an effort to compute the maximum outflow rate $\left(R_{\dot{m}}^{\max }\right)$ that is being originated from the disc. While doing so, for a given $a_{k}$, we calculate $R_{\dot{m}}^{\max }$ in terms of viscosity parameter $(\alpha)$ by varying the remaining inflow parameters independently. The obtained result is depicted in Fig. 8 where we show the variation of maximum outflow rates $R_{\dot{m}}^{\max }$ with $\alpha$. In the figure, solid, dashed and dotted curves denote the results corresponding to $a_{k}=0.0$ (black), 0.4 (red) and 0.8 (blue), respectively. We find that $R_{\dot{m}}^{\max }$ initially increases gradually with $\alpha$ and reaches to its maximum value and finally starts decreasing with the increasing $\alpha$ as shown in the figure. We observe that for a given $\alpha, R_{\dot{m}}^{\max }$ weakly correlates with $a_{k}$ which seems to be consistent with the results of inviscid flow as reported in Aktar et al. (2015). Overall, we find that the maximum outflow rate typically lies in the range $3 \% \lesssim R_{\dot{m}}^{\max } \lesssim 19 \%$ for widespread viscosity parameter $(\alpha)$.

\subsection{Effect of cooling on outflow rates}

Until now, we present all the result in the advection dominated regime i.e., $f=1$. In this section, we discuss the effect of cooling on outflow rates around rotating black hole. Here, we consider the parametric cooling as prescribed by Narayan \& Yi (1994) for our analysis. In Fig. 9, we plot the variation of shock locations $\left(x_{s}\right)$ (upper panel), outflow rates $\left(R_{\dot{m}}\right)$ (middle panel) and compression ratio $(R)$ (lower panel) with cooling factor $f$. To obtain the result, here we choose $x_{\text {inj }}=500, \mathcal{E}_{\text {inj }}=0.0015, \alpha=0.05$ and $a_{k}=0.5$, respectively. In the figure, solid, dotted and dashed curves represent the results corresponding to the angular momentum at $x_{\text {inj }}$ as $\lambda_{\text {inj }}=3.975,4.0$ and 4.025, respectively. In Fig. 9a, we find that the shock location proceeds towards the black hole horizon with the decrease of cooling parameter $(f)$. Actually, in presence of cooling, flow loses its energy while accreting towards the black hole. In particular, cooling is more effective in the post-shock region compared to the pre-shock region due to the enhanced density and temperature distributions. This reduces the post-shock thermal pressure which eventually compels the shock front to move towards the black hole in order to maintain pressure balance across the shock. As a consequence, the size of the PSC is decreased with the increase of cooling causing the reduction of $R_{\dot{m}}$ as depicted in Fig. 9b. In addition, as the shock front moves closer to the black hole, the corresponding compression ratio $(R)$ is enhanced with cooling as shown in Fig. 9c. We also observe that for a fixed cooling parameter, the shock location recedes away from black hole with the increase of angular momentum at the outer edge $\left(x_{\mathrm{inj}}\right)$. This provides an indication that the shock transition seems to be centrifugally driven.

We continue our study of shock parameter space in the $\mathcal{E}_{\text {in }}-\lambda_{\text {in }}$ plane in presence of cooling. Towards this, we examine the modification of the parameter space with the increase of the cooling factor $f$ and present it in Fig. 10. Here, we choose $a_{k}=0.5$ and $\alpha=0.05$, respectively. As before, the region bounded by the solid and dotted curves represent the 

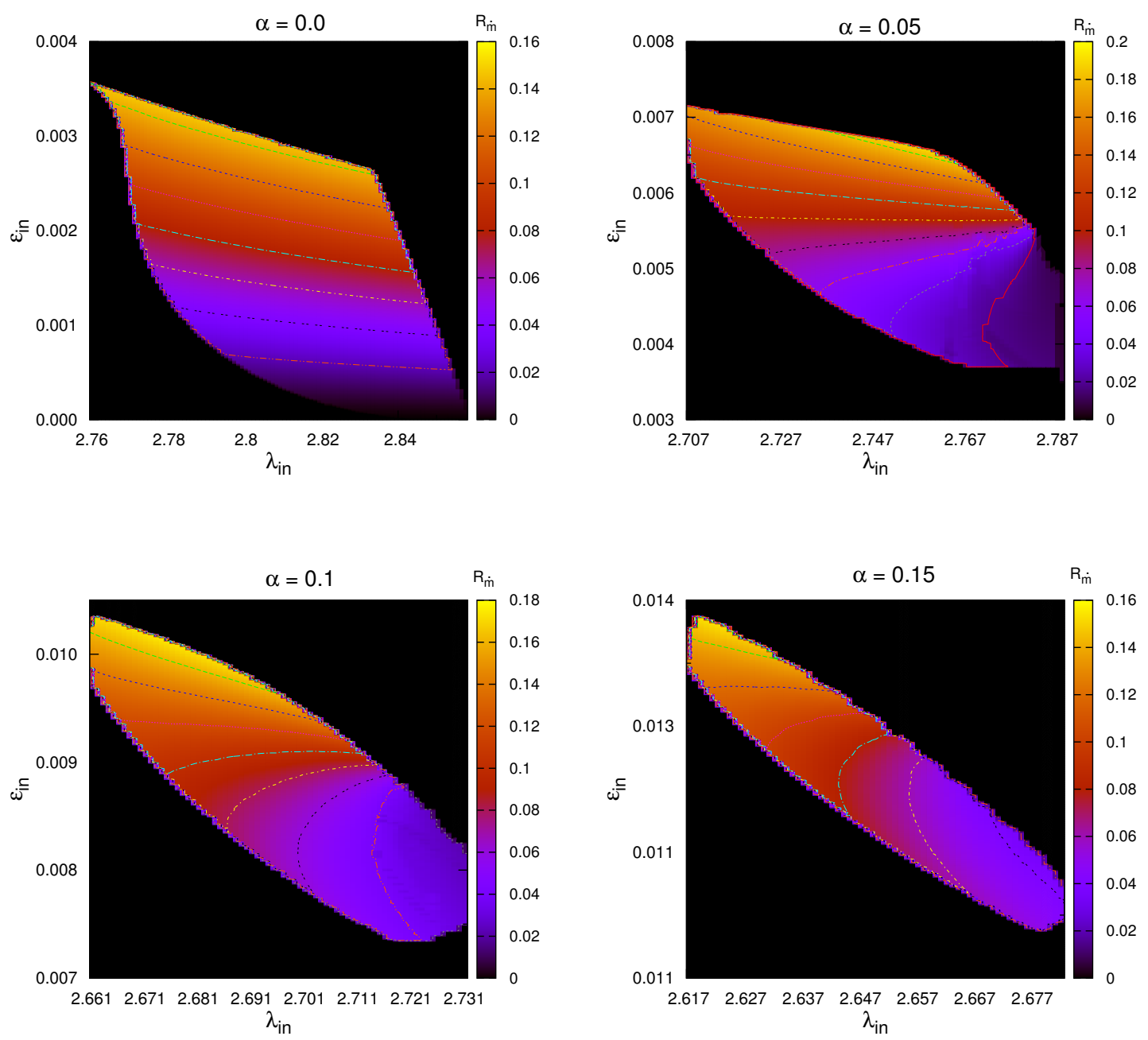

Figure 7. Two dimensional (2D) surface projection of three dimensional (3D) plot of flow energy $\left(\mathcal{E}_{\text {in }}\right)$, angular momentum $\left(\lambda_{\text {in }}\right)$ and outflow rates $\left(R_{\dot{m}}\right)$. In each panel, vertical color coded bar represents the estimated range of outflow rates. Here, the viscosity parameters are chosen as $\alpha=0.0,0.05,0.1$ and 0.15 , respectively and we fix black hole spin as $a_{k}=0.5$. See text for details.

shock parameter space in absence and in presence of mass loss. In this figure, chosen cooling factors $(f)$ are marked. As the cooling is increased, the effective region of the shock parameter spaces both with and without mass loss is reduced and it is shifted towards the negative energy region. Needless to mention that beyond a critical cooling limit, namely $f \rightarrow 0.05$, parameter space for outflow disappears completely.

\subsection{Application to source GRO J1655-40}

In this section, we attempt to constrain the rotation parameter i.e., spin of astrophysical black holes based on our accretion-ejection formalism. For the purpose of representation, we consider a Galactic black hole source GRO J1655-40 which is transient in nature and known to produce superluminal jets (Hjellming \& Rupen 1995, Tingay et al. 1995 Zhang et al. 1997). The source is considered to be one of the nearest Galactic black hole source with a distance of $3.2 \mathrm{kpc}$
(Migliari et al. 2007) and inclination angle $69 \pm 2$ (González Hernández et al. 2008). So far, it has been observed that this source exhibits the maximum QPO frequency $(\sim 450 \mathrm{~Hz})$ among all the known black hole candidates (Remillard et al. 2002 Belloni et al. 2012). The mass of the source is believed to be well constrained by dynamical method that estimates the range of mass to be $5.1 M_{\odot}$ to $6.3 M_{\odot}$ (Greene et al. 2001 Beer \& Podsiadlowski 2002). However, the measurement of the spin of the black hole remains inconclusive yet. Numerous groups used various methods while predicting the spin of the black hole with large uncertainties, namely the spectral continuum model predicts the spin to be in the range of 0.65 to 0.75 (Shafee et al. 2006), whereas Miller et al. (2009) predicted the spin to be in the range of 0.94 to 0.98 using relativistic reflection and disk continuum emission models. However, recent measurement which is based on the relativistic precession model of low frequency QPOs obtains the spin to be $\sim 0.29$ (Motta et al. 2014). Meanwhile, in the theoretical front, efforts were made to model the origin of 


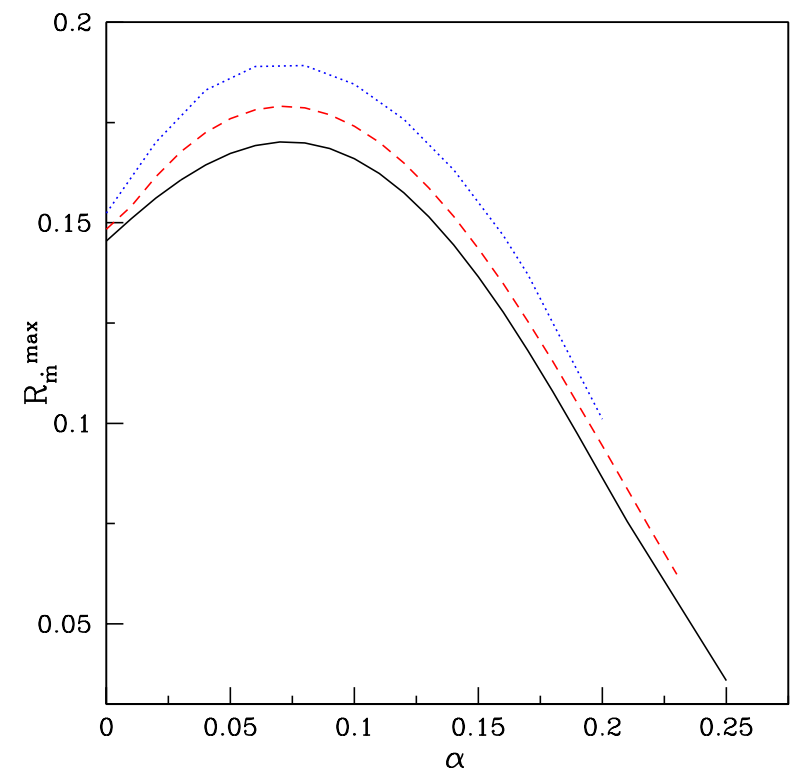

Figure 8. General behaviour of maximum outflow rates $R_{\dot{m}}^{\max }$ as function of viscosity parameter $\alpha$. Solid, dashed and dotted curves are for $a_{k}=0.0$ (black), 0.4 (red) and 0.8 (blue), respectively. See text for details.

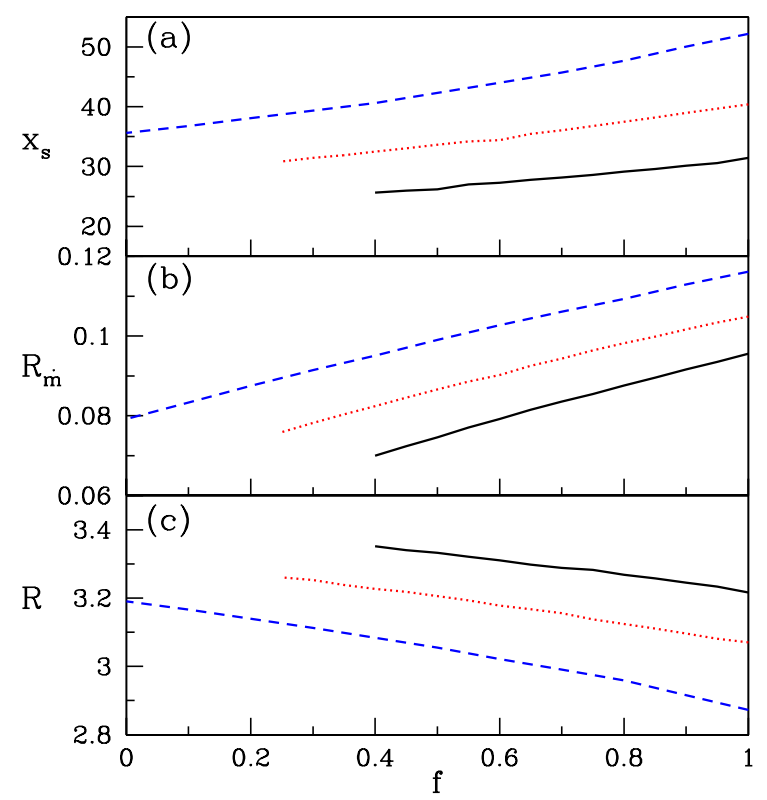

Figure 9. Plot of shock locations $\left(x_{s}\right)$ (upper panel), outflow rates $\left(R_{\dot{m}}\right)$ (middle panel) and compression ratio $(R)$ (lower panel) as function of the cooling factor $f$. Solid, dotted and dashed curves denote the results for $\lambda_{\text {inj }}=3.975$ (black), 4.0 (red) and 4.025 (blue), respectively. Here, we fix the outer boundary at $x_{\text {inj }}=500$ and flow energy as $\mathcal{E}_{\mathrm{inj}}=0.0015$. The viscosity parameter and spin values are chosen as $\alpha=0.05$ and $a_{k}=0.5$, respectively.

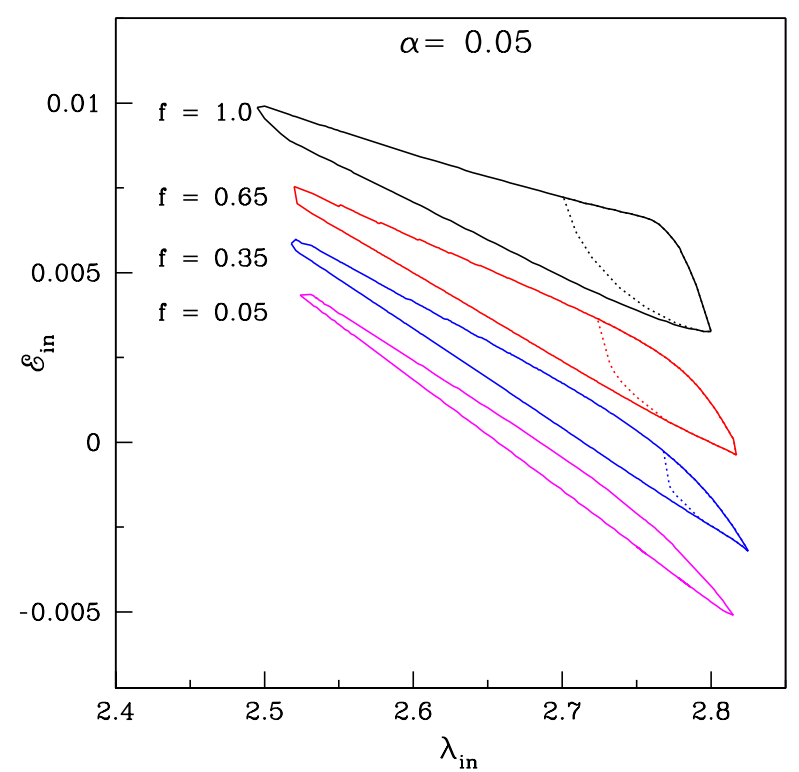

Figure 10. Comparision of shock parameter spaces obtained for various cooling parameters. Region bounded by the solid curve is for without mass loss case and the region bounded by the dotted curve represents results with mass loss case. Cooling parameters are marked in the figure. Here, we consider $\alpha=0.05$ and $a_{k}=0.5$. See text for details.

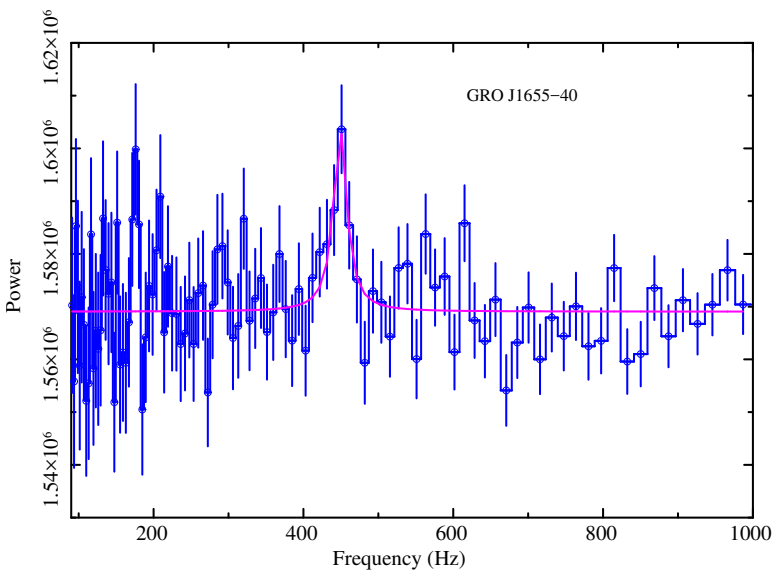

Figure 11. Power Density Spectrum (PDS) of the source GRO J1655-40 observed on MJD 50335.9, showing the HFQPO signature of frequency $(\sim 450 \mathrm{~Hz})$. See text for details.

the high frequency QPOs considering the coupling between the angular momentum of the black hole (i.e., spin) and orbital frequency at the innermost stable circular orbit (ISCO) and obtain the range of black hole spin as $0.2<a_{k}<0.67$ (Abramowicz \& Kluz̀niak 2001). A very recent work carried out by Stuchlík \& Koloś (2016) predicted the lower limit of the spin to be $>0.3$ based on the non-geodesic string loop oscillation model of twin high frequency QPOs. Since the discrepancy of the spin measurement is not settled yet, this motivates us to constrain the rotation parameter based on our formalism.

In this analysis, we choose the maximum observed QPO 


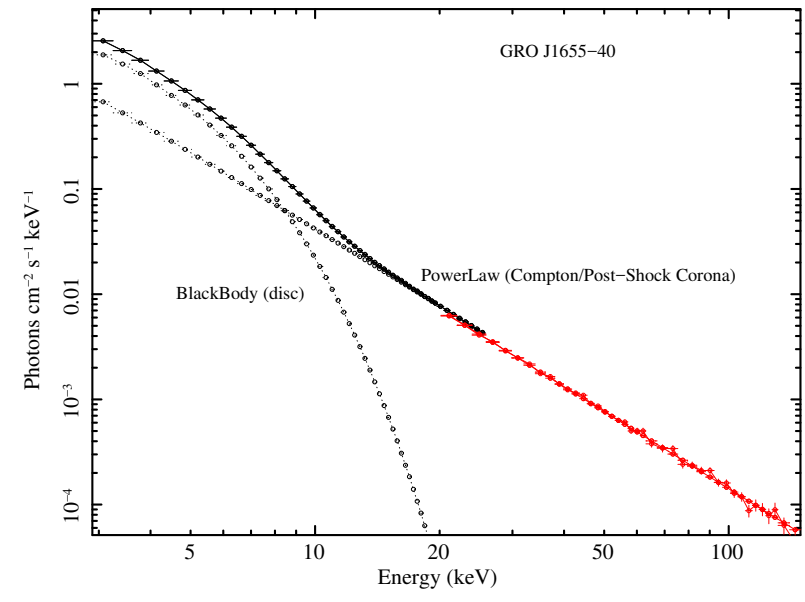

Figure 12. X-ray energy spectrum of the black hole source GRO J1655-40 observed on MJD 50335.9. Spectrum is fitted with the phenomenological models consisting of diskbb and powerlaw components. See text for details.

frequency $(\sim 450 \mathrm{~Hz})$ for the source as a parameter $\left(\nu_{Q P O}^{\max }\right)$ in order to constrain the spin of GRO J1655-40. Subsequently, we consider one of the observations of the 1996 outburst data of the source in order to extract the HFQPO feature and its X-ray spectral nature. We used the archival data of RXTE satellite observed on MJD 50335.9 ( $09^{\text {th }}$ September of 1996) with typical exposure of $\sim 4 \mathrm{ksec}$ (Remillard et al. 2002, Belloni et al. 2012). Standard techniques for data reduction and analysis are followed for temporal and spectral analysis of $R X T E$ data. We extract the lightcurve using the event mode data with a time resolution of $0.38 \mathrm{msec}$. This lightcurve was used to generate the power spectrum with 4096 newbins and with a geometrical binning factor of -1.02. Since we are interested to search for HFQPOs, we consider only the frequency range of $100 \mathrm{~Hz}$ to $1000 \mathrm{~Hz}$. Fig. 11 shows the power spectrum plotted in the Lehay powerFrequency space. The model used to fit the power spectrum consisted of a constant and a Lorentzian. The best-fitted model parameters are HFQPO $\sim 449 \mathrm{~Hz}, \mathrm{FWHM} \sim 21 \mathrm{~Hz}$ with $\chi_{\text {red }}^{2}$ of $0.88\left(\chi^{2} /\right.$ dof $\left.=85.5 / 94\right)$. We extract the energy spectrum of the same observation using the standard method/techniques (Radhika et al. 2016a) from the PCA (3-25 keV) and the HEXTE (20-150 keV). The combined spectrum (3 -150 keV) was modelled using a phenomenological model of diskbb and powerlaw for the thermal emission from the disk and Comptonized component (i.e., inner part of the disk, here PSC) respectively. While modeling, we kept fixed $n H \sim 0.7 \times 10^{22}$ (using Dickey \& Lockman (DL) method $^{2}$ ) and included an absorption edge around $4 \mathrm{keV}$. Fig. 12 shows the un-folded energy spectrum with the model components. The best-fitted model parameters are $T_{\text {in }} \sim 1.32 \mathrm{keV}, N_{\text {diskbb }} \sim 967$, photon index $(\alpha) \sim$ 2.43 and $N_{p o} \sim 11.42$ with $\chi_{r e d}^{2}$ of $0.74\left(\chi^{2} /\right.$ dof $\left.=64.7 / 87\right)$. Spectral parameters indicate that the source was in thermally dominated spectral state with strong emission at high energy. Therefore, the possible 'accretion' scenario perhaps could be that the disk is very close to the black hole with a

\footnotetext{
${ }^{2}$ https://heasarc.gsfc.nasa.gov/cgi-bin/Tools/w3nh/w3nh.pl
}

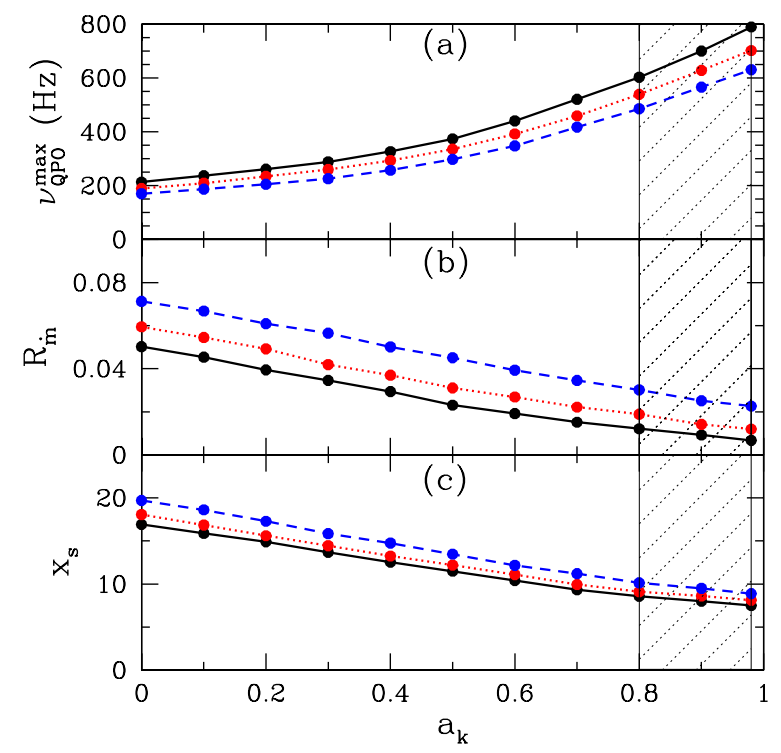

Figure 13. Variation of (a) maximum QPO frequency $\left(\nu_{Q P O}^{\max }\right)$, (b) corresponding mass outflow rates $\left(R_{\dot{m}}\right)$ and (c) shock location $\left(x_{s}\right)$ with the spin $\left(a_{k}\right)$ corresponding to the black hole mass $\left(M_{\mathrm{BH}}=6 M_{\odot}\right)$ of GRO J1655-40. Solid, dotted and dashed curves represent $\alpha=0.01$ (black), 0.05 (red) and 0.1 (blue), respectively. In each panel, shaded region denotes results for $a_{k}>0.8$. See text for details.

compact corona (i.e., PSC) to produce strong thermal emission (disk contribution) at low energy and power-law distribution (Comptonised contribution) at high energies (see Remillard \& McClintock (2006)). Usually, as the post-shock flow remains hot and dense compared to the pre-shock flow, the cooling rate in the post-shock disc becomes high. Interestingly, when the infall time in the post-shock region is comparable to the cooling time scale, shock starts to oscillate (Molteni et al. 1996b). In an another effort, Das et al. (2014) carried out hydrodynamical numerical simulations of black hole accretion disk and showed that when viscosity exceeds its critical value, post-shock disc exhibits oscillatory behaviour. Irrespective to the origin, if PSC starts to oscillate, the source demonstrates QPOs (Lee et al. 2011 Nandi \begin{tabular}{|l|l|l|l|}
\hline et al. 2012, Suková \& Jianuk 2015). Similarly, when the &
\end{tabular} compact corona exhibits rapid modulation, the source may display high frequency QPOs.

Now, we calculate the QPO frequency $\left(\nu_{Q P O}\right)$ based on our accretion-ejection model. Here, we compute the infall time from the post-shock velocity profile as $t_{\text {infall }}=$ $\int d t=\int_{x_{s}}^{x_{i n}} \frac{d x}{v(x)}$, where $v(x)$ is the post-shock velocity. The integration is carried out from the shock location to the inner sonic point as the distance between the inner sonic point and event horizon is negligibly small. We estimate the QPO frequency as $\nu_{Q P O}=\frac{1}{t_{Q P O}} \sim \frac{1}{t_{\text {infall }}}$ in units of $\frac{r_{g}}{c}$ (Molteni et al. 1996b). Accordingly, QPO frequency is converted into the unit of Hertz when it is multiplied with $\frac{c}{r_{g}}$. Here, we consider the mass of the source GRO J1655-40 as $6 M_{\odot}$. With this, we calculate the maximum QPO frequency $\left(\nu_{\mathrm{QPO}}^{\max }\right)$ as function of $a_{k}$ for various viscosity parameters as depicted in Fig. 13a. Here, we vary the remaining flow variables $\left(i . e ., \mathcal{E}_{\text {in }}\right.$ and $\left.\lambda_{\text {in }}\right)$ freely to obtain $\nu_{\mathrm{QPO}}^{\max }$. Filled circles 
connected with the solid, dotted and dashed curves denote the results corresponding to $\alpha=0.01$ (black), 0.05 (red) and 0.1 (blue), respectively. In the present analysis, when $a_{k}$ is increased, shock usually forms closer to the black hole (see Fig. 3) which apparently yields the high frequency QPOs. Therefore, a positive correlation between $\nu_{\mathrm{QPO}}^{\max }$ and $a_{k}$ is very much desirable and it is seen in Fig. 13a. Moreover, we find that the maximum QPO frequency generally lies in the range $150 \mathrm{~Hz} \lesssim \nu_{Q P O}^{\max } \lesssim 780 \mathrm{~Hz}$ depending on the values of $a_{k}$ and $\alpha$ for this source. The variation of the outflow rates $\left(R_{\dot{m}}\right)$ corresponding to the results displayed in the upper panel of Fig. 13 is shown in the middle panel (Fig. 13b). As $\nu_{Q P O}^{\max }$ corresponds to the minimum shock location $\left(x_{s}^{\min }\right)$ and $\nu_{Q P O}^{\max }$ increases with the increase of $a_{k}$, the size of PSC (which is basically the area of the jet base) is consequently reduced resulting the lowering of outflow rate. For completeness, we also show the variation of shock location associated to $\nu_{Q P O}^{\max }$ in the bottom panel (Fig. 13c). In each panel, the shaded region represents the results obtained for $a_{k} \geqslant 0.8$.

Since the observed maximum QPO frequency $\left(\nu_{Q P O}^{\max }\right)$ of the source GRO J1655-40 is found as $\sim 450 \mathrm{~Hz}$, in our subsequent analysis, we investigate the ranges of black hole spin parameter $\left(a_{k}\right)$ and viscosity parameter $(\alpha)$ that provide $\nu_{Q P O}^{\max } \sim 450 \mathrm{~Hz}$. In Fig. 14, we plot $a_{k}$ along the $\mathrm{x}$-axis and $\alpha$ along the $\mathrm{y}$-axis and identify the region that renders the $\nu_{Q P O}^{\max }$ for this source. Based on our model calculation, we observe that the spin parameter of the source under consideration has the value $a_{k} \geqslant 0.57$ which seems to be in agreement with the observational results of Shafee et al. (2006) and Miller et al. (2009). In addition, a partial agreement is also seen between our estimated range of spin value and the result reported by Abramowicz \& Kluz̀niak (2001). In addition, we find that the allowed range of $\alpha$ for this source is restricted below $\alpha^{\max } \sim 0.18$. The correlation study between the spin parameters of other black hole sources and their observed HFQPOs is in progress and will be reported elsewhere.

\section{CONCLUDING REMARKS}

In this paper, we describe a self-consistent formalism to calculate the mass outflow rates from the dissipative accretion flow around the rotating black holes in presence of viscosity and cooling processes. Effort of this kind was not considered before to investigate the outflow properties around rotating black holes. We present the governing equations for accretion and ejection mechanisms in details and demonstrate the methodology to obtain the inflow-outflow solutions. Since the black hole accretion solutions are transonic in nature, we present the sonic point analysis and demonstrate the sonic point properties. With this, we obtain the complete global accretion solutions that may contain shock wave. Because of shock compression, an excess thermal pressure is developed across the shock that causes the post-shock flow to become puffed up which equivalently behaves like Comptonized corona (i.e., PSC). A part of the inflowing matter after being intercepted at PSC is diverted along the black hole rotation axis to form thermally driven bipolar outflow (Molteni et al. 1996). Considering this appealing mechanism, subsequently, we obtain the coupled inflow-outflow solutions. We show that for a wide range of inflow parameters,

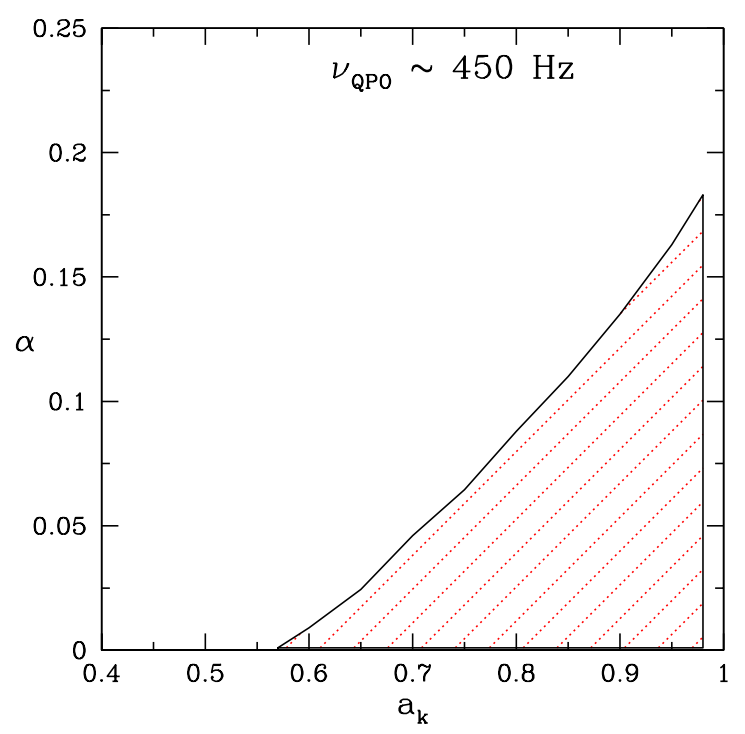

Figure 14. Identification of the ranges of viscosity parameter $(\alpha)$ and black hole spin $\left(a_{k}\right)$ that cater HFQPO frequency $\nu_{Q P O} \sim$ $450 \mathrm{~Hz}$ observed in GRO J1655-40. See text for details.

accretion flow can exhibit outflows even when the viscosity and cooling are very high. We observe that shocks in accretion flow form within the range of few to several tens of Schwarzschild radius depending on the inflow parameters. We also observe that as the effect of dissipation is increased, namely in the form of viscosity and cooling processes, shock moves closer to the horizon and the corresponding outflow rate $\left(R_{\dot{m}}\right)$ correlates with the shock location for flows with same outer boundary condition (Fig. 3).

We show that outflows from the dissipative accretion disc can occur around non-rotating as well as rotating black holes. When a part of the inflowing matter is ejected out from the PSC as outflow, the post-shock pressure is decreased that eventually allows the shock front to proceed towards the horizon to maintain the pressure balance across the shock. Interestingly, in absence of mass loss $\left(R_{\dot{m}}=0\right)$, when the triggering of shock transition takes place at its minimum location from the horizon $\left(x_{s}^{\min }\right)$, the coupled inflow-outflow solution ceases to exist as the stationary shock conditions are not satisfied there. This eventually indicates that when the effects of viscosity and cooling remain fixed, the range of the inflow parameters for outflow solutions is reduced compared to the global shocked accretion solution with $R_{\dot{m}}=0$. Effectively, the domain of the shock parameter space spanned by $\lambda_{\text {in }}$ and $\mathcal{E}_{\text {in }}$ is shrunk with the increase of dissipation and it is reduced further when massloss is included (Fig. 5). In this study, we observe three distinct features of the modified shock parameter space. We find that when viscosity parameter $(\alpha)$ is increased keeping spin of the black hole $\left(a_{k}\right)$ and cooling parameter $(f)$ fixed, the effective area of the shock parameter space, both in absence and presence of mass loss, shifts to higher energy and lower angular momentum domain. This happens due to the fact that the accreting matter becomes relatively much hotter when it enters into the gravitational potential of rapidly spinning black hole compared to the non-rotating black hole 


\section{Ramiz Aktar, Santabrata Das, Anuj Nandi, H. Sreehari}

(Kumar \& Chattopadhyay 2014). We further compare the shock parameter spaces for flows with increasing viscosity parameter keeping cooling parameter $(f)$ and $a_{k}$ fixed. Due to increase of viscosity, accreting matter not only transports more and more angular momentum outward, but it also causes the flow to become hotter. This evidently renders the shock parameter space to shift to the higher energy and lower angular momentum domain (Fig. 5). For a cooling dominated flow, the shock parameter space is shrunk and shifted to negative energy region (Fig. 10).

We estimate the maximum viscosity parameter that provides global shocked accretion solution both by excluding mass loss $\left(\alpha_{n o}^{\max }\right)$ and including mass loss $\left(\alpha_{o}^{\max }\right)$. Since the possibility of shock formation reduces in presence of mass loss, we obtain $\alpha_{o}^{\max }<\alpha_{n o}^{\max }$ for a given $a_{k}$. We further find that the obtained values of $\alpha_{n_{o}}^{\max } \sim 0.35$ and $\alpha_{o}^{\max } \sim 0.25$ in the limit of $a_{k} \rightarrow 0$ are in agreement with the results reported by Chakrabarti \& Das (2004) and Kumar \& Chattopadhyay (2013). Moreover, we calculate the maximum mass outflow rate $\left(R_{\dot{m}}^{\max }\right)$ as function of viscosity parameter $(\alpha)$ by exploring all possible combination of inflow parameters and find that $R_{\dot{m}}^{\max }$ is largely obtained for higher $\mathcal{E}_{\text {in }}$ and lower $\lambda_{\text {in }}$ values irrespective to the values of $a_{k}$ (Fig. 7). In Fig. 8, we depict the computed values of $R_{\dot{m}}^{\max }$ as function of $\alpha$ and find the range as $3 \% \leqslant R_{\dot{m}}^{\max } \leqslant 19 \%$.

It is worthy to address a very interesting finding that is emerged out from our study here. We have pointed out that when the viscosity as well as cooling parameters are considered beyond their critical values, coupled inflow-outflow solution ceases to exist. However, non-steady shock still may present as shown by Ryu et al. (1997); Das et al. (2014). Meanwhile, Molteni et al. (1996b); Chakrabarti \& Manickam (2000) showed that QPO frequency of emergent hard radiation from the black holes is proportional to the infall time of the accreting matter. Adopting this prescription, we employ our formalism to calculate the maximum QPO frequency $\left(\nu_{\mathrm{QPO}}^{\max }\right)$ as function of $a_{k}$ for flows with various $\alpha$. We then attempt to constrain the spin of the black hole source GRO J1655-40 considering the highest QPO frequency $\sim 450 \mathrm{~Hz}$ observed in this source (Fig. 11). Based on our present analysis, we find that $a_{k} \geqslant 0.57$ for this source (Fig. 14).

An important point is to be noted that in late 90's, Blandford \& Begelman (1999) found the advection dominated inflow-outflow solutions (ADIOS) around a Newtonian central object. There are subtle differences between our study and ADIOS, one of which arises mainly due to the choice of the accretion rate equation. In ADIOS model, the accretion rate is assumed to satisfy the power low dependence on radial coordinate as $\dot{M} \propto x^{s}$ with $0 \leqslant s<1$ whereas no such restriction is imposed in our study (see equation (3)). In addition, in this work, we have considered dissipative accretion flow including viscosity and radiative cooling which were ignored in ADIOS model. Moreover, we obtain the self-consistent accretion-ejection solutions that are coupled via Rankine-Hugoniot shock and in ADIOS model, mass loss is allowed to take place from all radial coordinates.

In this work, the relativistic effects of the central black hole are taken in to account by adopting the pseudo-Kerr gravitational potential (Chakrabarti \& Mondal 2006). This potential satisfactorily describes the general relativistic effect around black hole for $a_{k} \leqslant 0.8$ and it allows us to investi- gate properties of coupled inflow-outflow solutions in a simpler way. However, we continue our study even for $a_{k}>0.8$ to oversee the overall properties of the inflow-outflow solutions. In addition, for simplicity, we consider the parametric cooling mechanism ignoring the physically motivated cooling processes. Moreover, we consider the constant adiabatic index to describe the accretion-ejection model instead of estimating it self-consistently based on the thermal properties of the flow. In this formalism, we have disregard the issues of the collimation of jets and its powering processes although magnetic fields may be an important ingredient to explain the transient relativistic jets and its collimation mechanism. Implementation of all such relevant issues are beyond the scope of the present paper. However, we argue that even with the above approximations, overall findings of our present analysis will remain qualitatively unaltered.

\section{ACKNOWLEDGMENTS}

Authors thank the anonymous referee for useful comments and constructive suggestions. AN thanks GD, SAG; DD, PDMSA and Director, ISAC for encouragement and continuous support to carry out this research. We thank Toru Okuda for discussion. This research has made use of the data obtained through High Energy Astrophysics Science Archive Research Center on-line service, provided by NASA/Goddard Space Flight Center.

\section{REFERENCES}

Abramowicz M. A., Kluz̀niak W., 2001, A\&A, 374, 19

Aktar R., Das, S., Nandi, A., 2015, MNRAS, 453, 3414

Beer M. E., Podsiadlowski P., 2002, MNRAS, 331, 351

Becker P. A., Kazanas D., 2001, ApJ, 546, 429

Belloni T. M., Sanna A., Mndez M., 2012, MNRAS, 426, 1701

Blandford R. D., Begelman M. C., 1999, MNRAS, 303, L1

Blandford R. D., Znajek R. I., 1977, MNRAS, 179, 433

Chakrabarti S. K., 1989, ApJ, 347, 365

Chakrabarti S. K., 1996a, ApJ, 464, 664

Chakrabarti S. K., 1996b, MNRAS, 283, 325

Chakrabarti S. K., 1996c, PhR, 266, 229

Chakrabarti S. K., 1999, A\&A, 351, 185

Chakrabarti S. K., Manickam S. G., 2000, ApJ, 531, 41

Chakrabarti S. K., Goldoni P., Wiita P. J., Nandi A., Das S., 2002, ApJ, 576, 45

Chakrabarti S. K., Das S., 2004, MNRAS, 349, 649

Chakrabarti S. K., Mondal S., 2006, MNRAS, 369, 976

Cheung C. C., 2002, ApJ, 581, 15

Chattopadhyay I., Das S., 2007, New Astron., 12, 454

Chattopadhyay I., Kumar R., 2016, MNRAS, 459, 3792

Corbel et al.., 2013, MNRAS, 428, 2500

Das S., et al., 2001a, A\&A, 379, 683

Das S., Chattopadhyay I., Chakrabarti S. K., 2001b, ApJ, 557,983

Das S., 2007, MNRAS, 376, 1659

Das S., Chakrabarti, S. K., 2008, MNRAS, 389, 371

Das S., Chattopadhyay I., 2008, New Astron., 549, 556

Das S., Becker P A., Le T., 2009, ApJ, 702, 649 
Das S., Chakrabarti S. K., Mondal S., 2010, MNRAS, 401, 2053

Das S., et al., 2014, MNRAS, 442, 251

Das S., Aktar R., 2015, BASI, 12, 47

De Villiers, J. P., et al., 2005, ApJ, 620, 878

Falcke H., Körding E., Markoff S., 2004, A\&A, 414, 895

Fender R. P., Belloni T. M., Gallo E., 2004, MNRAS, 355, 1105

Fender R. P., Homan J., Belloni T. M., 2009, MNRAS, 396, 1370

Fender R. P., Gallo E., 2014, Space Sci. Rev., 183, 323

Fender R., Munoz-Darias T., 2016, Lecture notes in Physics, Berlin Springer Verlag, 905, 65

Feroci M., et al., 1999, A\&A, 351, 985

Ferrari A., 1998, ARA\&A, 36, 539

Frank I., King A. R., Raine D., 2002, Accretion power in Astrophysics (Cambridge)

Fukue J., 1987, PASJ, 39, 309

Fukumura K., Tsuruta S., 2004, ApJ, 611, 964

Gallo E., Fender R. P., Pooley G. G., 2003, MNRAS, 344, 60

Giri K., Chakrabarti S. K., Samanta M. M., Ryu, D., 2010, MNRAS, 403, 516

Greene J., Bailyn C. D., Orosz J. A., 2001, ApJ, 554, 1290

González Hernández J. I., Rebolo R., Israelian G., 2008, A\&A, 478, 203

Junor W., Biretta J. A., Livio M., 1999, Nature, 401, 891

Koide S., et al., 2002, Science, 295, 1688

Kumar R., Chattopadhyay I., 2013, MNRAS, 430, 386

Kumar R., Chattopadhyay I., 2014, MNRAS, 443, 3444

Hjellming R. M., Rupen M. P., 1995, Nature, 375, 464

Lee S. J., Ryu D., Chattopadhyay I., 2011, ApJ, 728, 142

Lu J. F., Gu W. M., Yuan F., 1999, ApJ, 523, 340

Machida M., Hayashi M. R., Matsumoto R., 2000, ApJ, 532,67

Matsumoto R., Kato S., Fukue J., \& Okazaki A. T., 1984, PASJ, 36, 71

McClintock J. E., Narayan R., Steiner J., 2014, Space Sci. Rev., 183, 295

McKinney J. C., Gammie C. F., 2004, ApJ, 611, 977

Mezcua M., et al., 2013, MNRAS, 436, 3128

Mezcua M., et al., 2015, MNRAS, 448, 1893

Migliari et al.., 2007, ApJ, 670, 610

Miller J M., Reynolds C S., Fabian A C., Miniutti G., Gallo L C., 2009, ApJ, 697, 900

Miller J. M., et al., 2012, ApJ, 757, 11

Miller J. M., et al., 2012, ApJL, 759, 6

Miller-Jones J. C. A., et al., 2012, MNRAS, 421, 468

Mirabel I. F., et al., 1992, Nature, 358, 215

Motta S E., Belloni T M., Stella L., Muǹoz-Darias T., Fender R., 2014, MNRAS, 437, 2554

Mirabel I. F., Rodriguez L. F., 1994, Nature, 371, 46

Mirabel I. F., Rodriguez L. F., 1998, Nature, 392, 673

Mirabel I. F., 2003, New A Rev., 47, 471

Molteni D., Lanzafame G., Chakrabarti S. K., 1994, ApJ, 425, 161

Molteni D., Ryu D., Chakrabarti S. K., 1996a, ApJ, 470, 460

Molteni D., Sponholz, H., Chakrabarti S. K., 1996b, ApJ, 457, 805

Mondal S.,Chakrabarti S. K., 2006, MNRAS, 371, 1418
Nandi A., Chakrabarti S. K., Vadawale S. V., Rao A. R., 2001, A\&A, 380, 245

Nandi A., Debnath D., Mandal S., Chakrabarti S. K., 2012, A\&A, 542, 56

Narayan R., Yi I., 1994, ApJ, 428, 13

Narayan R., McClintock J. E., 2012, MNRAS, 419, 69

Okuda T., 2014, MNRAS, 441, 2354

Okuda T., Das S., 2015, MNRAS, 453, 147

Paczyński B., Wiita P., 1980, A\&A, 88, 23

Penrose R., 1969, Nuovo Cimento Rivista Serie, 1, 252

Radhika D., Nandi A., 2014, AdSpR, 54, 1678

Radhika D., Nandi A., Agrawal V. K., Seetha S., 2016a, MNRAS, 460, 4403

Radhika D., Nandi A., Agrawal V K., Mandal S., 2016b, MNRAS, 462, 1834

Remillard R. A., Muno M. P., McClintock J. E., Orosz J. A., 2002, ApJ, 580, 1030

Remillard R. A., McClintock J. E., 2006, ARA\&A, 44, 49

Russell D. M., Gallo E., Fender R. P., 2013, MNRAS, 431, 405

Ryu D., Chakrabarti S. K., Molteni D., 1997, ApJ, 474, 378

Sarkar B., Das S., 2016, MNRAS, 461, 983

Shafee et al., 2006, ApJ, 636, 113

Shakura N. I., Sunyaev R. A.,1973 A\&A, 24, 337.

Steiner J. F., McClintock J. E., Narayan R., 2013, ApJ, 762,104

Stuchlík Z., Koloś M., 2016, ApJ, 825, 13

Suková P., Janiuk A., 2015, MNRAS, 447, 1565

Tingay et al.., 1995, Nature,374, 141

Vadawale S. V., Rao A. R., Nandi A., Chakrabarti S. K., 2001, A\&A, 370, 17

Zhang et al., 1997, ApJ, 479, 381 